\title{
Fractonic Chern-Simons and BF theories
}

\author{
Yizhi You $\odot,{ }^{1}$ Trithep Devakul, ${ }^{2}$ S. L. Sondhi, ${ }^{2}$ and F. J. Burnell ${ }^{3}$ \\ ${ }^{1}$ Princeton Center for Theoretical Science, Princeton University, Princeton, New Jersey 08544, USA \\ ${ }^{2}$ Department of Physics, Princeton University, Princeton, New Jersey 08544, USA \\ ${ }^{3}$ Department of Physics, University of Minnesota Twin Cities, Minneapolis, Minnesota 55455, USA
}

(Received 17 July 2019; accepted 27 November 2019; published 29 May 2020)

\begin{abstract}
Fracton order is an intriguing new type of order which shares many common features with topological order, such as topology-dependent ground-state degeneracies, and excitations with mutual statistics. However, it also has several distinctive geometrical aspects, such as excitations with restricted mobility, which naturally lead to effective descriptions in terms of higher-rank gauge fields. In this paper, we investigate possible effective field theories for three-dimensional fracton order, by presenting a general philosophy whereby topological-like actions for such higher-rank gauge fields can be constructed. Our approach draws inspiration from Chern-Simons and BF theories in $2+1$ dimensions, and imposes constraints binding higher-rank gauge charge to higher-rank gauge flux. We show that the resulting fractonic Chern-Simons and BF theories reproduce many of the interesting features of their familiar two-dimensional cousins. We analyze one example of the resulting fractonic Chern-Simons theory in detail, and show that upon quantization it realizes a gapped fracton order with quasiparticle excitations that are mobile only along a subset of one-dimensional lines, and display a form of fractional self-statistics. The ground-state degeneracy of this theory is both topology and geometry dependent, scaling exponentially with the linear system size when the model is placed on a three-dimensional torus. By studying the resulting quantum theory on the lattice, we show that it describes a $\mathbb{Z}_{s}$ generalization of the Chamon code.
\end{abstract}

DOI: 10.1103/PhysRevResearch.2.023249

\section{INTRODUCTION}

Topological quantum field theories (TQFTs) have been a powerful tool in developing our understanding of the possible strongly interacting, gapped phases of matter. In particular, they exhibit behaviors not perturbatively accessible from either weakly interacting or semiclassical limits, in which particles interact statistically and systems exhibit a ground-state degeneracy that depends on the topology of the underlying spatial manifold. This behavior, known as topological order, has drawn a tremendous amount of interest, and our understanding of where it may manifest itself in nature [1-4], its interplay with symmetry [5-8], and possible applications to quantum computing $[9,10]$ have developed rapidly in recent years.

Recently, a new class of phases, known as fractonic phases, have been discovered in the context of exactly solvable lattice models [11-18]. Fractonic phases [10-12,14-48] exhibit behaviors in some respects similar topological order, such as robust ground-state degeneracies, and statistical interactions between pointlike quasiparticles. However, they are also qualitatively different from topologically ordered phases in several respects: the ground-state degeneracy is not topological, but rather sensitive to geometric aspects such as

Published by the American Physical Society under the terms of the Creative Commons Attribution 4.0 International license. Further distribution of this work must maintain attribution to the author(s) and the published article's title, journal citation, and DOI. system sizes and aspect ratios, and excitations are generally subdimensional, meaning that they are either immobile, or that their motion is restricted to lines or planes in a threedimensional (3D) system.

Given the power of topological quantum field theory to study topological orders in two dimensions (2D), it is natural to ask whether there is a class of quantum field theories which captures fractonic behavior. Clearly, such field theories must be both similar to, and qualitatively different from, TQFTs. Specifically, a TQFT describes an infrared limit, the topological scaling limit, in which the details of the underlying lattice (or regularization) are unimportant, and universal topological physics emerges. Interestingly, the lattice also recedes in another scaling limit, this time near critical points, whose properties are famously captured by universal critical field theories. Fracton models, however, do not have a continuum limit in this strong sense: their ground-state degeneracies depend explicitly on the lattice size, and the subdimensional mobility of their excitations means that rotational symmetry can never emerge at long wavelengths. Finding a quantum field theory appropriate to describing fracton phases thus represents an interesting theoretical challenge.

A number of possible approaches to this challenge have been discussed in the literature thus far [16,19,21,25$31,34,41,49]$. For certain models, a connection to a continuous field theory can be made via a Higgs transition [21,25,26,29], though a continuum version of the action describing the infrared fixed point of these theories is not known in general $[25,28,40-42,45]$. Moreover, this formulation only describes a subset of the known fracton models; other models, such as the Chamon code [14,15], do not admit a Higgs-type description. 
A second approach is to work directly from a known fracton lattice model, and derive the constraints that it must impose on a resulting gauge theory. Once these constraints are known, often they can be imposed via a gauge-invariant continuum action, leading to actions reminiscent of $(2+1) \mathrm{D}$ BF theory. Drawing inspiration from similar results in twodimensional topologically ordered systems [50], Ref. [21] used this approach to derive a BF-like continuum field theory for the X-cube model [12]. A more general framework, relating constraints in excitations' mobility to generalized Gauss' laws, was developed by Ref. [31]. Finally, Gromov [49] has recently proposed a framework within which "matter" fields which exhibit a set of multipole conservation laws stemming from polynomial shift symmetries can be gauged to obtain fracton models.

In this work, we adopt a different approach, by "generalizing" $(2+1) \mathrm{D}$ TQFT's to the context of higher-rank gauge theories with a single time component $A_{0}$ (so-called "scalar" charge theories). That is, rather than considering usual vector gauge fields, we seek possible TQFT-like actions for tensor gauge fields, whose spatial gauge transformations may involve products of two derivatives. ${ }^{1}$ This is a natural choice if one desires to replicate some features of fractonic phases of matter [25,27,31,42,45,46,51-54].

Our main focus is on what we will call fractonic ChernSimons theory. Specifically, we will take as our starting point an action inspired by $(2+1) \mathrm{D}$ Chern-Simons theory, which imposes a constraint binding charge to the flux of a higherrank gauge field. We also comment briefly on the possibility of similar theories inspired by $(2+1) \mathrm{D} \mathrm{BF}$ theory (or mutual Chern-Simons theory), which are particularly interesting in the context of general higher-rank gauge theories, whose gauge transformations can contain mixed first- and secondorder polynomials in derivatives [31,49]. In both cases, we restrict our attention to Abelian [U(1)] theories, which are technically simpler to deal with than their non-Abelian counterparts.

The behavior of the resulting theories depends sensitively on the number of gauge fields present, since in a scalar charge theory our construction gives only a single Chern-Simons constraint. We will primarily discuss a gapped field theory that emerges naturally when we require our rank-2 gauge fields to transform in representations of $C_{3}$ rotations about a fixed $(1,1,1)$ axis. Since the appropriate representations are two dimensional, this leads to a theory with two spatial gauge fields, whose single propagating degree of freedom can be eliminated by our Chern-Simons constraint, leading to a fully gapped theory.

We discuss in detail both a continuum classical version of this model and a lattice-regularized quantum version. At the classical level, we find a theory whose gauge transformations imply that charged excitations (lineons) are mobile along only discrete sets of lines, and identify nonlocal (Wilson-line-like) gauge-invariant observables exhibiting a strong sensitivity to both the topology and the geometry of the spatial manifold. In

\footnotetext{
${ }^{1}$ More generally, the term "higher-rank gauge theory" refers to any symmetric gauge structure whose gauge transformation contains higher-order differential forms.
}

particular, we show that though imposing the Chern-Simons constraint does reduce the number of independent Wilson operators, this number grows with the linear system size.

Strikingly, upon quantizing our theory, we find that it has all of the expected hallmarks of type I fracton order. Specifically, it has a ground-state degeneracy that is sensitive to both the topology (periodic boundary conditions are required) and the geometry (aspect ratios and system sizes) of the system. Furthermore, its lineon excitations have nontrivial statistical interactions of the type exhibited in certain fractonic lattice models [31-34,55], in which pairs of particles propagating along different lines in the same plane may have mutual statistics. In fact, we show that by first quantizing this theory on a lattice, and then applying the Chern-Simons constraint, we are naturally led to a lattice Hamiltonian that can be viewed as a $\mathbb{Z}_{s}$ generalization of the Chamon code [14].

We also discuss an analog of Maxwell-Chern Simons theory model with three spatial gauge fields, corresponding to the off-diagonal elements of a symmetric rank-2 tensor. In this case, the single Chern-Simons constraint is insufficient to fully gap the theory. One interesting feature of this model is that in the absence of the Chern-Simons term it has been shown to be necessarily confined [56], whereas with our higher-rank Chern-Simons term confinement is suppressed and we find a deconfined $\mathrm{U}(1)$ phase with dipolar excitations mobile in two-dimensional planes.

Our approach highlights that, although our fractonic Chern-Simons theories are clearly not TQFTs, it is possible to construct field theories for higher-rank gauge fields that share several important features of the chiral $(2+1) D$ Chern-Simons theories. First, our fractonic Chern-Simons term creates selfstatistical interactions between charged excitations. Second, our fractonic Chern-Simons action is gauge invariant only up to a boundary term, implying that their boundaries host gapless surface states that cannot be realized in two dimensions with subsystem symmetry. These are closely related to the surface states of subsystem-symmetry-protected models described in Ref. [34].

The presence of such anomalous surfaces is surprising in light of the correspondence between our field theories and exactly solvable lattice models, which is not expected for systems with topologically protected gapless boundary modes. This is one of several hints that the regularization may play a more fundamental role in quantizing our higher-rank ChernSimons theories than it does for TQFTs or critical theories. Indeed, it is not clear whether it is possible to construct a well-defined continuum version of our compact $U(1)$ theory that correctly captures the low-energy behavior of the lattice model.

The paper is organized as follows. In Sec. II, we introduce a general formulation for Chern-Simons-type actions appropriate to models with three-component gauge fields $\left(A_{0}, A_{1}, A_{2}\right)$ and a single scalar charge. This formulation applies both to vector gauge theories, whose gauge transformations are linear in derivatives, and tensor gauge theories whose gauge transformations are quadratic in derivatives. In Sec. III, we discuss a particular realization of such a rank-2 theory, with two spatial gauge fields transforming under $C_{3}$ rotations about the $(1,1,1)$ direction. We discuss the possible gauge-invariant operators in this case, and show that the associated quadratic 
(in derivatives) gauge transformations lead to matter fields that are restricted to move on lines, and gauge-invariant "cage-net" operators similar to those previously discussed in the context of lattice fracton models [34,36,57].

In Sec. IV, we scrutinize the classical Chern-Simons theory of this rank-2 theory. In particular, we show that the ChernSimons constraint fixes all gauge-invariant operators except noncontractible loop operators, and discuss the number of independent loop operators of this type for the 3-torus. We also show that the Chern-Simons action is gauge invariant only up to a boundary term, and discuss the nature of the resulting boundary theory.

Section $\mathrm{V}$ describes a lattice regularization of our rank-2 gauge theory, which we use to discuss two distinct routes to quantization. In Sec. VI, we discuss quantizing the constrained lattice model, derive the resulting ground-state degeneracy on the $L \times L \times L$ torus, and describe the self-statistics and mutual statistics that follow from our Chern-Simons action. In Sec. VII, we first quantize the lattice gauge fields, and then impose the Chern-Simons constraint. We see that this leads to a lattice Hamiltonian that is a $\mathbb{Z}_{s}$ generalization of the Chamon code [14]. Finally, in Sec. VIII, we discuss adding a Chern-Simons term to the Maxwell action of a symmetric tensor gauge theory with four components, $\left(A_{0}, A_{x y}, A_{x z}, A_{y z}\right)$. We argue that though the resulting theory is gapless, it is nonetheless interesting as the Chern-Simons term appears to overcome the theory's expected confinement [56] in a manner very similar to the case of compact U(1) Maxwell-ChernSimons theory in $2+1$ dimensions [58].

\section{GENERAL HIGHER-RANK CHERN-SIMONS GAUGE THEORIES}

Our starting point is a theory with two spatial gauge fields $A_{1}$ and $A_{2}$, which will allow us to obtain a fully gapped Chern-Simons theory with a single constraint. Consider gauge transformations of the form

$$
A_{1} \rightarrow A_{1}+D_{1} \alpha, A_{2} \rightarrow A_{2}+D_{2} \alpha,
$$

where $D_{1}$ and $D_{2}$ are differential operators, whose form we will leave unspecified for now. Since we only have two gauge fields, the magnetic field defined has a single component

$$
B=D_{2} A_{1}-D_{1} A_{2} \text {. }
$$

Note that the magnetic field (2) is always gauge invariant; however, it is not necessarily the most relevant gauge-invariant magnetic field that we can write. If $D_{1}$ and $D_{2}$ share a common factor $\partial_{\ell}$, the operator $\partial_{\ell}^{-1} B$ is also gauge invariant. Throughout the paper, we will focus on the cases where $D_{1}, D_{2}$ do not have common factor and the lowest-order gauge-invariant term is the magnetic flux.

The gauge-invariant electric fields have the form

$$
E_{i}=\partial_{t} A_{i}-D_{i} A_{0},
$$

where we have introduced the usual time component of the gauge field, which transforms as

$$
A_{0} \rightarrow A_{0}+\partial_{t} \alpha
$$

under gauge transformations.
The generalized Chern-Simons action we consider is

$$
\mathcal{L}_{\mathrm{CS}}=\frac{s}{4 \pi}\left[A_{1} E_{2}-A_{2} E_{1}-(-1)^{\eta} A_{0} B\right],
$$

where $\eta=1$ if $D_{i}$ contain only even numbers of derivatives, and $\eta=2$ if they contain only odd numbers of derivatives. Under gauge transformations, we have

$$
\begin{aligned}
\delta \mathcal{L}_{\mathrm{CS}}= & \frac{s}{4 \pi}\left[D_{1} \alpha E_{2}-D_{2} \alpha E_{1}-(-1)^{\eta} \partial_{t} \alpha B\right] \\
= & \frac{s}{4 \pi}\left(D_{1} \alpha \partial_{t} A_{2}+(-1)^{\eta} \partial_{t} \alpha D_{1} A_{2}\right. \\
& -\left[D_{2} \alpha \partial_{t} A_{1}+(-1)^{\eta} \partial_{t} \alpha D_{2} A_{1}\right] \\
& \left.+D_{2} \alpha D_{1} A_{0}-D_{1} \alpha D_{2} A_{0}\right) .
\end{aligned}
$$

In the absence of boundaries, we may freely integrate by parts, to obtain

$$
\delta \mathcal{L}_{\mathrm{CS} ; \text { Bulk }}=0 \text {. }
$$

The boundary terms in general do not vanish, implying the existence of gapless boundary modes, whose precise nature depends on the choice of $D_{i}$. We will return to this point later when we discuss specific examples.

Irrespective of the choice of $D_{i}$, the Chern-Simons action (5) has several commonalities with the standard vector ChernSimons theory in $2+1$ dimensions. First, in the absence of sources the constraint simply sets $B=0$. Since there is only one component of the magnetic field, this one constraint is sufficient to eliminate the possibility of any propagating gauge degrees of freedom, leading to a gapped theory whose physics is entirely determined by operators describing pure gauge degrees of freedom. ${ }^{2}$ In ordinary Chern-Simons theory these are the holonomies, or gauge-invariant Wilson lines along noncontractible curves. We will discus the analog of Wilsonline operators for specific examples of $D_{i}$ in detail presently; these have the general form $e^{i \int_{s} A_{i}}$ with the submanifold $s$ chosen to ensure the operator is gauge invariant.

Second, irrespective of the choice of $D_{i}$, the gauge fields $A_{1}$ and $A_{2}$ are canonically conjugate. If both gauge fields are compact, this implies that a generalized Wilson operator of the form $e^{i \int_{s} A_{i}}$ must be discrete as well as compact. Thus, each of the generalized Wilson operators can take on only a finite, discrete set of values, which fully specify the states allowed in the absence of sources. On closed manifolds this can give either a finite or a countable ground-state degeneracy.

Finally, in the presence of matter fields, the Chern-Simons action (5) has the effect of binding charge to flux. To see this, we add matter fields to our Chern-Simons action in the standard way, by adding a term

$$
\mathcal{L}_{\text {Matter }}=A_{0} \rho-A_{i} J^{i},
$$

where the currents obey the conservation law

$$
D_{i} J^{i}=\partial_{t} \rho .
$$

\footnotetext{
${ }^{2}$ This only applies to the case where $D_{1}, D_{2}$ do not share any common factor. Otherwise, even the magnetic flux fluctuation is fixed, there might exist some local operator with lower order exhibiting a dispersive gapless mode.
} 
Depending on the specific form of the differential operator $D_{i}$, the theory might contain additional subsystem chargeconservation law and charge multipole conservation [49]. In the presence of sources the Chern-Simons constraint is

$$
B=D_{2} A_{1}-D_{1} A_{2}=\frac{2 \pi}{s} \rho
$$

which binds the generalized magnetic flux to charge. One might anticipate that a generalized Aharonov-Bohm effect may endow these charge-flux bound states with fractional statistics. Indeed, as gauge-invariant operators involving $A_{1}$ do not commute with gauge-invariant operators involving $A_{2}$, we will usually find at least some excitations with nontrivial mutual statistics.

However, as we will see, the choice of $D_{i}$ does have profound implications for the final theory, and is key to determining the nature and mobility of the sources, as well as the ground-state degeneracy. This is because it is the form of $D_{i}$, and not the action, that determines the gauge-invariant operators and conservation laws, which play an essential role in both of these physical properties. We therefore now discuss a few examples in detail.

\section{A. Example 1: $D_{1}$ and $D_{2}$ are linear in derivatives: Stacking of 2D Chern-Simons theory}

As a warmup, we consider the case where $D_{1}$ and $D_{2}$ are linear in derivatives. In this case, we can always write $D_{1}=$ $\partial_{l_{1}}, D_{2}=\partial_{l_{2}}$, with $l_{1}, l_{2}$ being two nonparallel directions. We will see that in two spatial dimensions this always yields the conventional 2D Chern-Simons theory, while in three spatial dimensions it behaves like a stack of decoupled Chern-Simons theories.

To understand this theory, let us first understand its symmetries. First, theories of this type will be rotationally invariant in the plane perpendicular to $l_{1}, l_{2}$. This is because $A_{i}$ transform like vectors under rotations in the $l_{1}, l_{2}$ plane. The gauge-invariant magnetic field $B$ is thus a scalar under in-plane rotations, as is the combination $A_{1} E_{2}-A_{2} E_{1}$. Thus, our Chern-Simons action is fully rotationally invariant within the $l_{1}, l_{2}$ planes. (Indeed, it is easy to check that in this case $\mathcal{L}_{\mathrm{CS}}$ has full Lorentz invariance.)

Second, the gauge transformations dictate that this theory has a conserved charge in each $2 \mathrm{D}$ plane. To see this, we couple our gauge fields to matter currents in the usual way:

$$
\mathcal{L}_{\text {Matter }}=A_{0} \rho-A_{1} J_{1}-A_{2} J_{2}
$$

Gauge invariance requires that the current is conserved, i.e.,

$$
\partial_{t} \rho=\partial_{l_{1}} J_{1}+\partial_{l_{2}} J_{2}
$$

If we integrate the right-hand side over any plane spanned by $\left(l_{1}, l_{2}\right)$ (in periodic boundary conditions), we obtain zero, implying charge conservation in each plane.

Next, let us examine the gauge-invariant operators. First, consider open line segments of the form $\int A_{1} d l_{1}, \int A_{2} d l_{2}$, where the lines run along the $\hat{l}_{1}$ and $\hat{l}_{2}$ directions, respectively.
Under gauge transformations we have

$$
\int_{x}^{y} A_{1} d l_{1} \rightarrow \int_{x}^{y} A_{1} d l_{1}+\left.\alpha\right|_{x} ^{y}, \quad \int_{x}^{y} A_{2} d l_{2} \rightarrow \int_{x}^{y} A_{2} d l_{2}+\left.\alpha\right|_{x} ^{y} .
$$

Thus, with periodic boundary conditions, closed lines of either type are gauge invariant. Further, we can see that a corner between a line along $\hat{l}_{2}$ and a line along $\hat{l}_{1}$ is gauge invariant. Thus, in addition to $E_{i}$ and $B$, there are also gauge-invariant contractible closed loops. Indeed, defining $\tilde{\overrightarrow{\vec{l}}}_{1}, \tilde{\overrightarrow{\vec{l}}}_{2}$ such that

$$
\tilde{\vec{l}}_{i} \cdot \vec{l}_{j}=\delta_{i j}
$$

we see that integrals of the form

$$
\oint\left(A_{1} d \tilde{l}_{1}+A_{2} d \tilde{l}_{2}\right)
$$

are gauge invariant for any closed curve in the $\left(l_{1}, l_{2}\right)$ plane.

Next, we examine how the Chern-Simons constraint $B=0$ restricts our possible choices of gauge-invariant operators. First, note that for a contractible closed curve bounding a region $\mathcal{R}$, we have

$$
\oint\left(A_{1} d \tilde{l}_{1}+A_{2} d \tilde{l}_{2}\right) \propto \int_{\mathcal{R}} B .
$$

(This can be shown by expressing $\tilde{l}_{i}, l_{i}$ in terms of a set of orthonormal basis vectors, and applying Stoke's theorem. Note that the coefficient of proportionality is not 1 unless $l_{i}$ and $l_{j}$ are orthogonal.) We conclude that the constraint $B=0$ ensures that all contractible Wilson-line operators are trivial.

Next, suppose we have periodic boundary conditions along the $l_{1}, l_{2}$ directions, such that there are also noncontractible gauge-invariant line operators. Since the two line operators concern lines in different directions, we will apply the ChernSimons constraint to each set of lines individually. With this logic, the Chern-Simons constraint then gives

$$
D_{2} \int_{1} A_{1}=0
$$

where $\int_{1}$ runs along a noncontractible curve in the $\hat{l}_{1}$ direction. In $2 \mathrm{D}$ this tells us that once we have fixed one $A_{1}$ line we have fixed them all; a similar argument applies for $A_{2}$. This is simply the familiar result that if there is no magnetic flux through the surface of the torus, the only degrees of freedom are the fluxes through its two noncontractible curves. In 3D we are free to choose one line in each $\left(l_{1}, l_{2}\right)$ plane, exactly as for a stack of decoupled Chern-Simons theories.

It is easy to see that quantizing such a theory also gives a result that is identical to a stack of decoupled Chern-Simons theories. Hence, we conclude that choosing $D_{1}, D_{2}$ to be linear in derivatives is essentially the same as choosing ordinary Chern-Simons theory in 2D, or a stack of ordinary ChernSimons theories in 3D.

\section{B. BF generalizations}

Before moving on to our second example, which will lead to a fractonic Chern-Simons theory whose behavior we will analyze in detail, it is worth pointing out that a similar generalization of mutual Chern-Simons, or BF, theories can 
be carried out. This generalization allows us to consider a wider variety of higher-rank gauge theories since, unlike the Chern-Simons construction described above, it can be applied to gauge fields whose gauge transformations are described by arbitrary polynomials in momenta. Gauge transformations of this type are necessary to capture current conservation laws arising from general subsystem symmetries [49], including type II fracton orders [31].

We begin with two gauge fields $\left(A_{0}, A_{1}, A_{2}\right)$ and $\left(B_{0}, B_{1}, B_{2}\right)$, which transform under gauge transformations according to

$$
\begin{array}{ll}
A_{0} \rightarrow A_{0}+\partial_{t} \alpha, & B_{0} \rightarrow B_{0}-\partial_{t} \alpha \\
A_{1} \rightarrow A_{1}+D_{1} \alpha, & B_{1} \rightarrow B_{1}+\tilde{D}_{1} \alpha, \\
A_{2} \rightarrow A_{2}+D_{2} \alpha, & B_{2} \rightarrow B_{2}+\tilde{D}_{2} \alpha,
\end{array}
$$

where

$$
D_{i}=D_{i}^{(e)}+D_{i}^{(o)}, \quad \tilde{D}_{i}=D_{i}^{(e)}-D_{i}^{(o)}
$$

with $D_{i}^{(e)}, D_{i}^{(o)}$ the differential polynomials containing even and odd numbers of derivatives, respectively. ${ }^{3}$ The higherrank $\mathrm{BF}$ action has the form

$$
\begin{aligned}
\mathcal{L}_{\mathrm{BF}}= & A_{0}\left(\tilde{D}_{1} B_{2}-\tilde{D}_{2} B_{1}\right)+A_{1}\left(\tilde{D}_{2} B_{0}+\partial_{t} B_{1}\right) \\
& +A_{2}\left(-\partial_{t} B_{1}-\tilde{D}_{1} B_{0}\right) .
\end{aligned}
$$

It is easy to check that this action is gauge invariant up to a boundary term. In the presence of sources, it imposes the constraints

$$
\begin{aligned}
& \left(\tilde{D}_{1} B_{2}-\tilde{D}_{2} B_{1}\right)=\rho_{A}, \\
& \left(D_{1} A_{2}-D_{2} A_{1}\right)=\rho_{B},
\end{aligned}
$$

where $\rho_{A}, \rho_{B}$ are the charges coupled to the $A$ and $B$ gauge fields, respectively. Provided $D_{1}, D_{2}$ does not share any common factor, these constraints are sufficient to eliminate any propagating modes, leading to a gapped theory describing a stable infrared fixed point. Further, it is clear that $A_{1}$ and $B_{2}$ (and $A_{2}$ and $B_{1}$ ) are canonically conjugate, such that the sources of $A$ and $B$ will acquire mutual statistics upon quantizing the theory. In this way, a wide variety of higherrank gapped fractonic actions can be constructed. We defer a discussion of the many interesting examples to future work, except to note that the BF-like field theory of the X-cube model proposed by Ref. [16] involves a construction of this type, albeit modified to work with gauge fields with three spatial components, whose gauge transformations share common factors.

\section{EXAMPLE II: FRACTONIC CHERN-SIMONS THEORY WITH DIPOLE EXCITATIONS}

Let us now consider an example that will lead to a fractonic Chern-Simons theory. To obtain this, we will take $D_{i}$ to be quadratic in derivatives. In this case (unlike for linear $D_{i}$ ),

${ }^{3}$ For general differential polynomial $D_{i}^{(e)}, D_{i}^{(o)}$, the coefficient in each differential term is dimensionful so such operator is only well defined on the lattice.

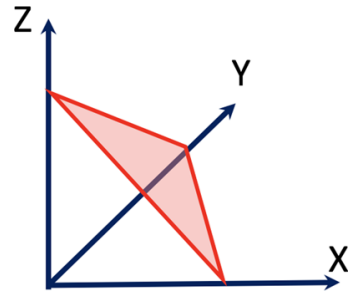

FIG. 1. Illustration of the $x, y, z$ and $u, v, w$ coordinates. The red plane is the 111 plane and the three boundary lines of the red triangle denote the $u, v, w$ directions. The $C_{3}$ rotation on the 111 plane rotates the red triangle by $2 \pi / 3$ and permutes the axis along $x, y, z$.

we have several choices, distinguished by their transformation under spatial symmetries.

For our example, consider a system with a cubic geometry, with the cubic axes $x, y$, and $z$. We will not require our action to have full cubic symmetry; indeed, we will see later that full cubic symmetry is compatible with our Chern-Simons action only for a specific choice of the coupling constant. Instead, we require invariance under $C_{3}$ rotations about the (111) direction. Thus, $D_{i}$ (and consequently $A_{i}$ ) must transform in an irreducible representation $\Gamma$ such that $\mathbf{1} \in \Gamma \otimes \Gamma$, to ensure that the quantities $B$ and $A_{1} E_{2}-A_{2} E_{1}$ transform as scalars under $C_{3}$ rotations. We will also require that the $D_{i}$ transform nontrivially under $C_{3}$ rotations; this ensures that both $A_{1}$ and $A_{2}$ are required to construct a symmetric action.

The full set of irreducible representations of $C_{3}$ are given in Appendix B. If $D_{i}$ are quadratic in derivatives, we have two choices for the irreducible representation $\Gamma$, which we denote $\Gamma^{a}$ and $\Gamma^{b}$ :

$$
\begin{aligned}
& D_{1}^{a}=\frac{1}{\sqrt{3}} \partial_{\ell} \partial_{u}, D_{2}^{a}=\frac{1}{\sqrt{3}} \partial_{\ell} \partial_{v}, \\
& D_{1}^{b}=\frac{\sqrt{2}}{\sqrt{3}} \partial_{u} \partial_{u_{\perp}}, D_{2}^{b}=\frac{1}{2 \sqrt{2}}\left(\partial_{u}^{2}-\partial_{u_{\perp}}^{2}\right)-\frac{1}{\sqrt{6}} \partial_{u} \partial_{u_{\perp}},
\end{aligned}
$$

where relative to the cubic axes $x, y, z$, we have defined (see Fig. 1)

$$
\begin{aligned}
& \hat{\ell}=\frac{1}{\sqrt{3}}(\hat{x}+\hat{y}+\hat{z}), \\
& \hat{u}=\frac{1}{\sqrt{2}}(\hat{y}-\hat{z}), \\
& v=\frac{1}{\sqrt{2}}(z-x), \\
& w=(-u-v)=\frac{1}{\sqrt{2}}(x-y)
\end{aligned}
$$

in terms of which $u_{\perp}$, the direction orthogonal to $u$, is

$$
\hat{u}_{\perp}=\frac{1}{\sqrt{3}}(-2 v-u)=\frac{1}{\sqrt{6}}(2 \hat{x}-\hat{y}-\hat{z}) .
$$

Under arbitrary rotations about the $\ell$ axis, $\Gamma^{a}$ transforms like a vector, with an angular momentum of 1 along the $\ell$ axis, while $\Gamma^{b}$ transforms like a rank-2 tensor, with angular momentum 2. For $C_{3}$ rotations, however, where the angular momentum 3 representation transforms like a scalar, these are effectively two vector representations. 
Thus, we have $1 \in \Gamma^{\alpha} \otimes \Gamma^{\beta}$ for all combinations of $\alpha, \beta$. Consequently, we may choose $D_{i}$ to be an arbitrary linear combination of $D_{i}^{a}$ and $D_{i}^{b}$. We will see later that, provided both irreducible representations appear with nonzero coefficients, the resulting theories are closely analogous. Thus, we will take

$$
\begin{aligned}
& D_{1}=D_{1}^{a}+D_{1}^{b}=\partial_{x} \partial_{u}=\frac{1}{\sqrt{2}} \partial_{x}\left(\partial_{y}-\partial_{z}\right), \\
& D_{2}=D_{2}^{a}+D_{2}^{b}=\partial_{y} \partial_{v}=\frac{1}{\sqrt{2}} \partial_{y}\left(\partial_{z}-\partial_{x}\right), \\
& -D_{1}-D_{2}=\partial_{z} \partial_{w}=\frac{1}{\sqrt{2}} \partial_{z}\left(\partial_{x}-\partial_{y}\right) .
\end{aligned}
$$

Under gauge transformations, we have

$$
\begin{aligned}
A_{1} & \rightarrow A_{1}+\partial_{x} \partial_{u} \alpha, \\
A_{2} & \rightarrow A_{2}+\partial_{y} \partial_{v} \alpha, \\
-A_{1}-A_{2} & \rightarrow-A_{1}-A_{2}+\partial_{z} \partial_{w} \alpha .
\end{aligned}
$$

The $C_{3}$ rotations about the $(1,1,1)$ direction permute $(u, v, w)$ and $(x, y, z)$ directions. Since $\alpha$ is a scalar, this implies that $C_{3}$ rotations permute $A_{1}, A_{2}$, and $-A_{1}-A_{2}$ (and similarly for $\left.E_{1}, E_{2}\right)$ :

$$
\begin{aligned}
C_{3}: x & \rightarrow y, y \rightarrow z, z \rightarrow x, \\
A_{1} & \rightarrow A_{2}, A_{2} \rightarrow-\left(A_{1}+A_{2}\right),-\left(A_{1}+A_{2}\right) \rightarrow A_{1}, \\
D_{1} & \rightarrow D_{2}, D_{2} \rightarrow-\left(D_{1}+D_{2}\right),-\left(D_{1}+D_{2}\right) \rightarrow D_{1} .
\end{aligned}
$$

The gauge-invariant magnetic field given in Eq. (2) is

$$
B=\left(\partial_{x} \partial_{u} A_{2}-\partial_{y} \partial_{v} A_{1}\right) .
$$

It is easy to check that $B$ (as well as the combination $A_{1} E_{2}-$ $A_{2} E_{1}$ ) transforms as a scalar under $C_{3}$ rotations, as it must if our theory is to be symmetric with $A_{0}$ rotationally invariant.

\section{A. Conservation laws}

Before studying Chern-Simons theory per se, let us understand what properties of the theory are dictated solely by the structure of its gauge transformation laws. The canonical coupling of gauge fields to sources

$$
\mathcal{L}_{\text {matter }}=A_{0} \rho-A_{1} J^{1}-A_{2} J^{2}
$$

implies the current conservation relation

$$
\partial_{t} \rho-D_{1} J_{1}-D_{2} J_{2}=0 .
$$

Note that in order for $\mathcal{L}_{\text {matter }}$ to be invariant under $C_{3}$ rotations, we must have

$$
C_{3}: J_{1} \rightarrow J_{2}-J_{1}, J_{2} \rightarrow-J_{1} .
$$

Evidently,

$$
\partial_{t} \int d^{3} \mathbf{r} \rho=\int d^{3} \mathbf{r}\left(D_{1} J_{1}+D_{2} J_{2}\right)=0
$$

so that charge is conserved in the system as a whole. Note that here we assume periodic boundary conditions and single valuedness of all currents, such that the integral of any derivative over all space is zero.
In addition, however, from Eq. (25) we see that both operators $D_{i}$ contain only terms with at least one derivative in each $u-v$ plane. Thus, we also have

$$
\partial_{t} \int d u d v \rho=0
$$

and charge is conserved in each $u-v$ plane. In addition, all terms in $D_{1}$ and $D_{2}$ contain either $\partial_{x}$ or $\partial_{y}$ (or both), such that the charge is conserved in each $x-y$ plane, and similarly for the $x-z$ and $y-z$ planes.

Finally, since charge conservation in an individual plane automatically implies that the dipole moment orthogonal to that plane is conserved, our theory has conserved dipole moments along the $x, y, z$, and $\ell$ (or $x+y+z$ ) directions. The net result of these four dipole conservation laws is that dipole mobility in our system is severely restricted. Consider a dipole oriented along the $z$ direction, which has a conserved dipole moment orthogonal to both the $(x, y)$ and the $(u, v)$ planes. As a consequence, it can move only along the line $x+y=0$, which lies in the intersection of these two planes. Similar considerations apply to other dipole orientations: all dipole excitations in this theory are restricted to move along lines.

\section{B. Gauge-invariant cage-net operator}

Having understood the theory's conservation laws, we now consider the nature of the gauge-invariant line operators. We will see that these reflect the one-dimensional motion of dipolar excitations, as well as introducing a cage-net structure similar to that noted in other fracton models [57].

To identify what types of operators we should study, observe that one obvious difference relative to the usual vector gauge theory is the dimension of the gauge field $A$ : if $\alpha$ is dimensionless [as is natural, since it appears as a U(1) phase rotation of the matter fields], then $A$ has mass dimension 2. Thus, dimensionless gauge-invariant operators in these theories require integrating along surfaces.

To determine which surfaces to examine, we will begin with dimensionful gauge-invariant line operators, whose end points transform as $\partial_{i} \alpha$ for some direction $i$. This implies that the end points of these open lines do not harbor charges, but instead are associated with the derivative of the charge along the $\hat{i}$ direction. To make physical sense of this, we define dimensionless gauge-invariant ribbon operators by integrating over a surface of width $a_{i}$ transverse to the line's orientation; the end points of such ribbons harbor a dipole oriented along the $\hat{i}$ direction. We will see presently that the associated dipole moments are conserved, suggesting that such a fixed length scale is not unnatural.

From the gauge transformations, we see that the theory admits six types of gauge-invariant line operators. Three of these are in-plane line operators:

$$
\Gamma_{u}=\int d u A_{1}, \quad \Gamma_{v}=\int d v A_{2}, \quad \Gamma_{w}=\int d w\left(-A_{1}-A_{2}\right),
$$


while three are line operators extending along the cubic axes $\hat{x}, \hat{y}, \hat{z}$.

$$
\Gamma_{x}=\int d x A_{1}, \quad \Gamma_{y}=\int d y A_{2}, \quad \Gamma_{z}=\int d z\left(-A_{1}-A_{2}\right) .
$$

The associated dimensionless gauge-invariant ribbon operators have the form

$$
W_{u}=e^{i \int_{x}^{x+a} \Gamma_{u} d x}, \quad W_{x}=e^{i \int_{u}^{u+\sqrt{2} a} \Gamma_{x} d u}
$$

and similarly for other pairs of directions. Here, $a$ is the fundamental dipole scale of our problem, as discussed above. Note that we choose the length of the fundamental dipoles in the $u, v, w$ directions to be $\sqrt{2}$ that of the fundamental dipoles along the $x, y, z$ directions. With this choice, a dipole along $u$ can be viewed as a combination of a dipole along $y$ and a dipole along $-z$.

Naively, the Wilson lines $\left\{\Gamma_{i}\right\}$ appear similar to line operators we would expect from a stack of $2 \mathrm{D}$ vector gauge theories discussed in the previous example. However, in the present theory the lines can run only along one of the six directions specified above (see Appendix A). Further, a corner between a $\Gamma_{u}$ line and a $\Gamma_{v}$ line must have a charge, by gauge invariance. To see this, consider the operator

$$
T=\int_{\vec{r}_{0}}^{\vec{r}_{1}} A_{1} d u+\int_{\vec{r}_{1}}^{\vec{r}_{2}} A_{2} d v+\int_{\vec{r}_{2}}^{\vec{r}_{0}}\left(-A_{1}-A_{2}\right) d w,
$$

where the points $\vec{r}_{0}, \vec{r}_{1}, \vec{r}_{2}$ form a triangle in one of the $(u, v)$ planes, with edges along the $u, v$, and $w$ directions respectively. For a usual rank-1 gauge theory, $T$ would be a gauge-invariant line operator. In our case, however, it is not: under gauge transformation, we find that

$$
T \rightarrow T+\left(\partial_{x}-\partial_{z}\right) \alpha\left(\vec{r}_{1}\right)+\left(\partial_{z}-\partial_{y}\right) \alpha\left(\vec{r}_{2}\right)+\left(\partial_{y}-\partial_{x}\right) \alpha\left(\vec{r}_{0}\right) .
$$

This shows that in order for $T$ to be gauge invariant, we must attach an infinitesimal dipole to each of the triangle's corners. Thus, though we do have Wilson-line-like operators in plane, these lines cannot bend without creating new dipolar excitations.

Similarly, lines running along the cubic axes cannot turn, either into other cubic directions or into the $(u, v)$ planes. However, because

$$
\left(\partial_{u}+\partial_{v}+\partial_{w}\right) \alpha=0,
$$

three lines running along orthogonal cubic axes can meet at a point without creating extra charges, as shown in Fig. 2. Similarly there is no charge at a trivalent vertex between $\sqrt{2} \Gamma_{y}, \Gamma_{u}$, and $\Gamma_{w}$ (and its appropriately rotated analogs) since

$$
\sqrt{2} \partial_{v} \alpha-\left(\partial_{x} \alpha-\partial_{y} \alpha\right)=0 .
$$

Note that Eq. (41) requires the lines to be correctly oriented at the vertex; the correct orientations are shown in Fig. 2.

From the above arguments, it is easy to see that similar gauge-invariant cage-net structures can be formed of our dimensionless Wilson ribbons (see Fig. 3), provided that we choose the dipole scales as specified in Eq. (37). The relative factor of $\sqrt{2}$ in the ribbons' widths ensures that the three
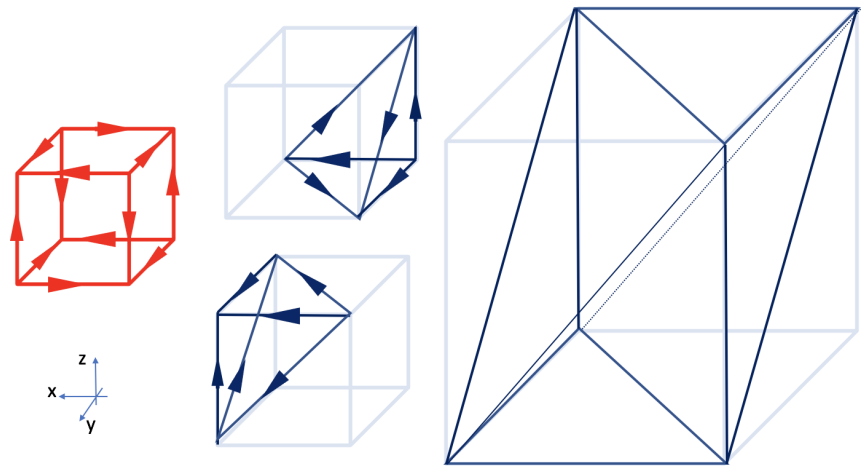

FIG. 2. Gauge-invariant cage nets of the symmetric rank-2 theory. With differential operators given in Eq. (25), the cages can have edges along the $x, y, z$ or $u, v, w$ directions.

dipoles can annihilate at the corresponding corners, so that the operators are gauge invariant.

In summary, the structure of the gauge transformations (25) leads to a qualitatively different fractonic Chern-Simons theory, in which the analog of the Wilson line is a gaugeinvariant dimensionless ribbon operator. These ribbons are not free to turn, but can meet at certain trivalent corners. This results in gauge-invariant "cage-net" operators, which can be tetrahedral, prismatic, or cubic as shown in Fig. 2.

In addition to cage nets we can also form gauge-invariant closed membranes by widening our ribbons to some multiple of the fundamental dipole scale, and then forming a closed surface along which all ribbons share an edge. In the extreme limit these are two-dimensional membrane operators that can extend in the $x-u, y-v$, or $z-w$ planes, and the associated cage nets are stretched into the diamond configuration shown in Fig. 3. We will not discuss these membrane operators in our analysis, however, since they can always be viewed as arrays of the fundamental ribbon operators $W_{\alpha}$ described above.

Finally, if we allow sources in our theory, open ribbon operators can also be gauge invariant, provided that we attach an appropriately oriented dipole at each end point (see Fig. 4).
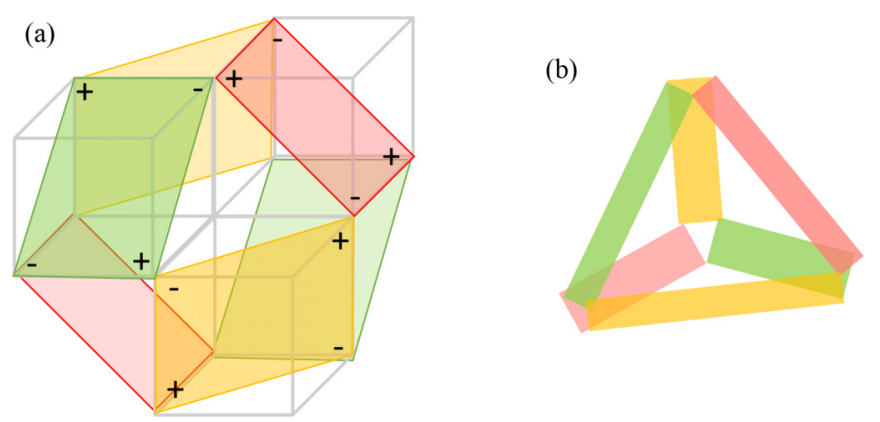

FIG. 3. (a) The red/yellow/green sheets represent thick ribbon operators. An isolated ribbon must harbor a charge at each corner; however, with appropriately oriented gauge fields these corner charges cancel in the configuration shown. (b) By lengthening and thinning the ribbons, we obtain a Wilson ribbon cage net. Note that the Wilson ribbon cage nets are gauge invariant only if we choose the width of ribbons along the $u, v, w$ directions to be longer by a factor of $\sqrt{2}$ than those along the $x, y, z$ directions. 

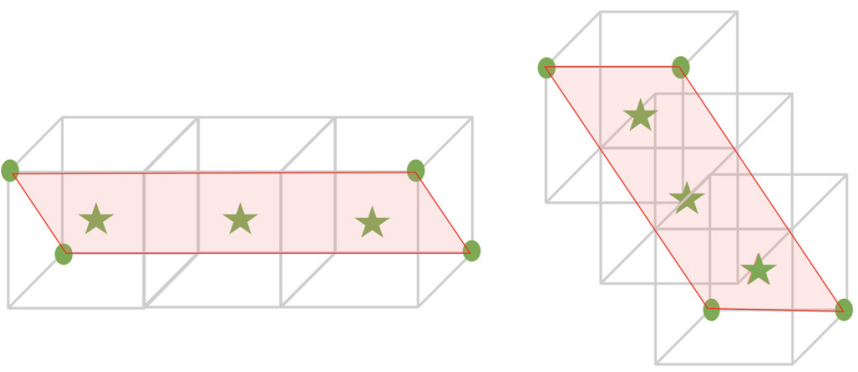

FIG. 4. Lineon excitation configurations: each end of an open Wilson ribbon extended along the $x$ direction must terminate on a dipole (or antidipole) with dipole moment oriented along $u$. Likewise, a Wilson ribbon extended along the $u$ direction hosts a dipole oriented along $x$ at each end point. Since Wilson ribbons cannot turn, these dipoles are mobile only along the direction of the ribbon, and are hence one-dimensional lineon excitations. Here, the star represents gauge fields $A_{1}, A_{2}$ while the dot represents charge $\rho$.

Each of the six possible line directions is thus associated with a dipolar source which, due to dipole conservation, is mobile only along a specific linear direction; these thus behave like the "lineons" typical of type I fracton theories. To strengthen the connection to fracton orders, we can also consider open membrane operators whose width is some multiple of the fundamental dipole scale. The charges appearing at the corners of each membrane are then immobile fractons.

\section{Related models with the same symmetry}

It is clear from the above discussion that (irrespective of our choice of action) a theory with the operators $D_{1}, D_{2}$ described above cannot be topological. In particular, the cagenet structure requires us to choose a fundamental dipole scale, and the theory is manifestly not scale invariant. The existence of this scale follows from the fact that the theory conserves dipole moment perpendicular to each individual $u-v, x-y, y-z$, and $x-z$ plane. Further, the structure of the cage nets is invariant only under a discrete set of $C_{3}$ rotations, which are naturally viewed as a subset of the rotational symmetries of the cubic lattice.

We may nonetheless ask whether related theories exist, which share the same $C_{3}$ rotation symmetry, and conserve dipole moment along four families of planes. To see that they do, we consider a more general form of the operators $D_{1}, D_{2}$ :

$$
D_{1}=\alpha D_{1}^{a}+\beta D_{1}^{b}, D_{2}=\alpha D_{2}^{a}+\beta D_{2}^{b},
$$

where $D_{i}^{a, b}$ are given in Eq. (22), and $\alpha, \beta$ are parameters which only take discrete values if we put the theory on the cubic lattice. Regardless of the choice of $\alpha, \beta$, the theory is $C_{3}$ rotation invariant; essentially, this is because both of the two-dimensional irreducible representations are vectorlike from the point of view of $C_{3}$ rotation symmetry.

For general nonzero $\alpha, \beta$, the differential operators have the explicit form

$$
\begin{aligned}
& D_{1} \sim\left[\partial_{x}+(\alpha-\beta) \partial_{\ell}\right]\left(\partial_{y}-\partial_{z}\right), \\
& D_{2} \sim\left[\partial_{y}+(\alpha-\beta) \partial_{\ell}\right]\left(\partial_{z}-\partial_{x}\right) .
\end{aligned}
$$
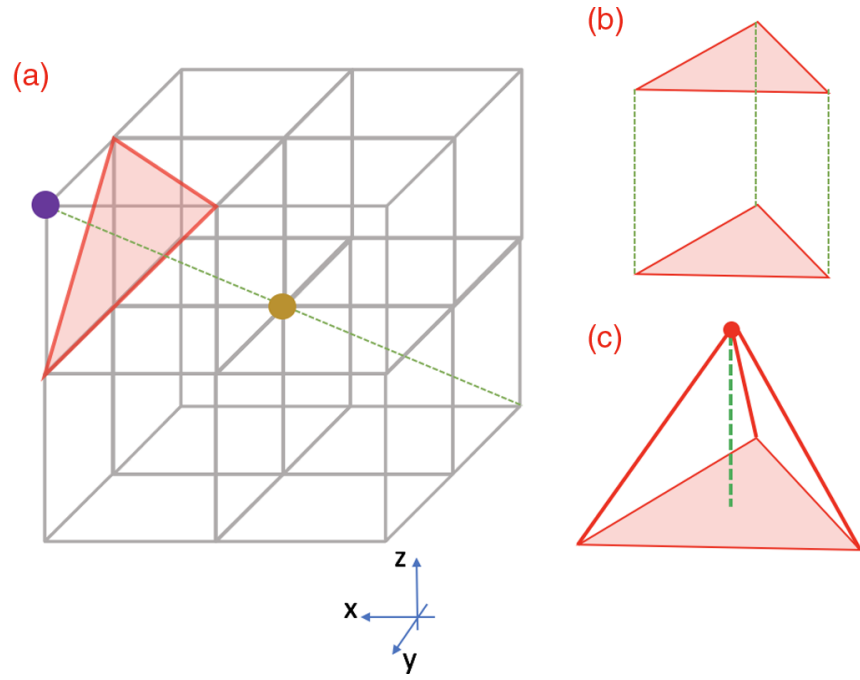

FIG. 5. By tuning $\alpha, \beta$ in Eq. (42), we are changing the height of the tetrahedron in our cage-net configuration. There are an infinite number of equivalent cage-net configurations related by this deformation. (c) A rotated view of the cage-net tetrahedron. The dimensions of the base of the tetrahedron (indicated with a shaded red triangle) are fixed, while its height along the (111) direction (represented by the green dotted line) varies depending on $\arctan (\alpha / \beta)$. (a) Examples of equivalent cage-net tetrahedra. The purple dot corresponds to the case where $\alpha, \beta=1$. The yellow dot corresponds to $\alpha=2, \beta=1$. (b) When $\beta=0$, the height of the tetrahedron goes to infinity and the cage-net configurations become a prism.

Thus, we obtain gauge-invariant ribbon operators extended along six nonparallel directions. At the end points of any open ribbon, there is a dipole. With an appropriate choice of the dipole scales in each direction, these six lines are allowed to meet at trivalent corners, leading to tetrahedral cage-net configurations as shown in Fig. 5. One face of the tetrahedron is an equilateral triangle in the $u-v$ plane; the remaining three have edges in the $x+(\alpha-\beta) \ell, y+(\alpha-\beta) \ell, z+(\alpha-\beta) \ell$ directions.

Following the arguments of Sec. III A, it is not hard to check that for general $\alpha$ and $\beta$, the charge is conserved in each $u-v$ plane, as well as in the other three families of planes spanned by two of the three vectors $x+(\alpha-\beta) \ell, y+$ $(\alpha-\beta) \ell, z+(\alpha-\beta) \ell$. These three planes are related by $C_{3}$ rotation along 111 axis. These conservation laws ensure that the dipoles described above can only move in one dimension. In particular, when $\alpha=2, \beta=1$, the cage-net configuration becomes a regular tetrahedron and the Chern-Simons coupling is odd under cubic rotation.

We note that the choices $\alpha=0$ and $\beta=0$ do lead to qualitatively different theories. Taking $\alpha=0$ clearly leads to a stack of two-dimensional theories since $D_{1}, D_{2}$ only involve derivatives in the $u-v$ plane. Taking $\beta=0$ gives $D_{1}=\partial_{l} \partial_{v}, D_{2}=\partial_{l} \partial_{u}$. As discussed in Appendix C, the line operators in the resulting theory are similar to those of a stack of ordinary 2D gauge theories, with both 2D particles mobile in each $u-v$ plane and a 1D particle mobile along the $\ell$ direction. 


\section{CLASSICAL CHERN-SIMONS THEORY OF $C_{3}$-SYMMETRIC RANK-2 MODEL}

Thus far, we have focused only characteristics of our field theory that result solely from the form of the operators $D_{1}, D_{2}$, which dictate the nature of the conservation laws, and the geometry of the gauge-invariant operators. We now turn to the implications of the Chern-Simons action. In the following sections, we will analyze this from a classical perspective, turning to the quantum theory in Sec. VI.

\section{A. Chern-Simons constraint}

Classically, the main role of the Chern-Simons Lagrangian (5) is to enforce the constraint

$$
B \equiv\left(\partial_{x} \partial_{u} A_{2}-\partial_{y} \partial_{v} A_{1}\right)=\frac{2 \pi}{s} \rho .
$$

This has two important effects on our classical theory: first, it fixes the value of all closed cage nets in our three-dimensional system. Second, it imposes conditions on parallel Wilson lines, drastically reducing the number of such operators that are independent.

First, we observe that the constraint $B=\frac{2 \pi}{s} \rho$ fixes the value of all cage-net operators. This can be checked directly by integrating the magnetic field over the volume enclosed by the cage net, which gives exactly the series of line operators associated with the cage net itself. A similar result holds for cage nets bounded by dimensionless ribbon operators. Hence, in the Chern-Simons theory, our gauge-invariant cage-net operators are constrained to take the value 0 .

Second, let us determine the effect of the Chern-Simons constraint on noncontractible Wilson operators. Consider operators of the type $W_{x}$, in the absence of sources. A priori there are $L_{y} L_{z} / a^{2}$ nonoverlapping operators of this type (where $a$ is the dipole scale), and similarly for other directions. However, integrating the constraint over $x$ gives

$$
\begin{aligned}
& 0=-\oint B d x=\partial_{z} \partial_{y} \oint d x A_{1}=\partial_{z} \partial_{y} \Gamma_{x}, \\
& 0=\oint B d y=\partial_{z} \partial_{x} \oint d y A_{2}=\partial_{z} \partial_{x} \Gamma_{y}, \\
& 0=\oint B d z=\partial_{x} \partial_{y} \oint d z\left(A_{1}+A_{2}\right)=-\partial_{x} \partial_{y} \Gamma_{z},
\end{aligned}
$$

where we have assumed periodic boundary conditions, and that $A$ is single valued. Thus, we may fix the value of $\Gamma_{x}$ (and hence $W_{x}$ ) along the boundaries of the $y z$ plane, by specifying one function of $y$ and one function of $z$, but having done this, the value of $\Gamma_{x}$ elsewhere in the plane is fixed. For an $L_{x} \times L_{y} \times L_{z}$ system this gives $\left(L_{y}+L_{z}\right) / a-1$ independent nonoverlapping ribbon operators $W_{x}$, and similarly for $W_{y}$. Further, once $\Gamma_{x}$ and $\Gamma_{y}$ are fixed everywhere, the condition that the cubic cage nets (see Fig. 2) must all be trivial then fixes $\Gamma_{z}$ along any line, such that the ribbon operators $W_{z}$ are fixed.

Similarly, we have

$$
0=\oint B d u=\partial_{v} \partial_{y} \oint d u A_{1}=\partial_{v} \partial_{y} \Gamma_{u} .
$$

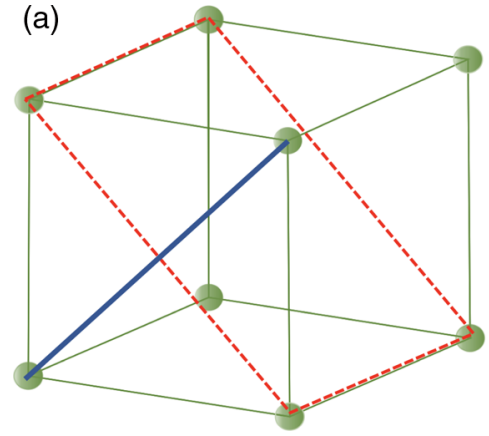

(b)

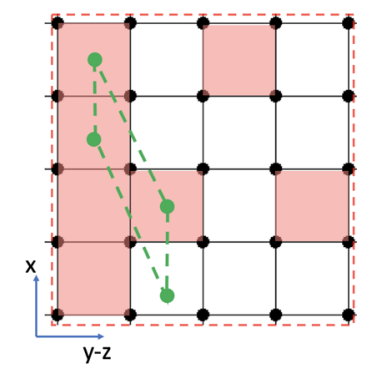

FIG. 6. Counting of independent nonoverlapping Wilson ribbons on an $L \times L \times L$ system. In (b), each square has side length $a$, representing the width of the fundamental Wilson ribbon; pink squares indicate the number of independent ribbon operators $W_{u}$ on an $(x, y+z)$ plane. The trajectories of the diagonal Wilson ribbons on a unit cell of the cubic lattice are illustrated in (a).

This again allows two types of solutions: either $\Gamma_{u}$ is constant in the $\hat{y}$ direction, or in the $\hat{v}$ direction (meaning that it satisfies $\left.\partial_{x} \Gamma_{u}=\partial_{z} \Gamma_{u}\right)$. Since

$$
\begin{aligned}
& \partial_{y}=\frac{1}{2}\left(\sqrt{2} \partial_{u}+\partial_{y}+\partial_{z}\right), \\
& \partial_{v}=-\frac{1}{2}\left(\partial_{u}+\sqrt{3} \partial_{u_{\perp}}\right),
\end{aligned}
$$

and $\partial_{u} \Gamma_{u}=0$, this effectively tells us that we may choose $\Gamma_{u}$ (and hence $W_{u}$ ) to be a function of $y+z$ or a function of $x$, but not both (Fig. 6 illustrates the relevant geometry). For $L_{i} \equiv L$ this naively gives another $2 L / a-1$ independent, nonoverlapping ribbon operators. However, not all of these can be independent of the $\Gamma_{x}$ since

$$
\oint \Gamma_{x} d u=\oint \Gamma_{u} d x .
$$

Thus, classically, on an $\mathrm{Na} \times \mathrm{Na} \times \mathrm{Na}$ system with periodic boundary conditions along the $x, y$, and $z$ directions, we anticipate $4 N-3$ independent line integrals for $A_{1}$, and the same number for $A_{2}$. Since the Chern-Simons constraint in the absence of sources requires that the cage-net configuration in Fig. 2 is trivial, the remaining line operators $\Gamma_{z}, \Gamma_{w}$ [containing integrals of $\left(A_{1}+A_{2}\right)$ ] are also fixed. Thus, there are $8 N-6$ independent global flux operators.

For general values of $L_{x}, L_{y}, L_{z}$ the counting of the number of independent line operators is more involved. For example, if the spatial lengths $L_{y} / a, L_{z} / a$ are coprime, a closed $\Gamma_{u}$ ribbon crosses every point in the $y-z$ plane; clearly, there is a maximum of one such operator for each $y-z$ plane, or a total of $L_{x}$ such operators, of which $L_{x}-1$ are independent of the $\Gamma_{x}$. This gives a total of $\left(L_{y}+L_{z}\right) / a+L_{x} / a-2$ independent line operators involving $A_{1}$. More generally, the number of lines along the $u$ direction in each $y$ - $z$ plane is given by $\operatorname{gcd}\left(L_{y} / a, L_{z} / a\right)$, and we obtain $\left(L_{y}+L_{z}\right) / a+L_{x} / a+$ $\operatorname{gcd}\left(L_{y} / a, L_{z} / a\right)-3$ independent line operators involving $A_{1}$. Similar considerations apply for line operators involving $A_{2}$.

Similarly, the number of independent nonoverlapping Wilson ribbons is sensitive to the boundary conditions since the noncontractible lines can go only along specific directions. Thus, twisting the boundary conditions can lead to dramatically different line operator counting. The dependence of the 
number of independent operators on both the aspect ratio and twist of the boundary conditions reflects the fact that our higher-rank Chern-Simons theory is not a topological field theory, but rather is sensitive to both the geometry and the topology of the system.

\section{B. Gauge invariance and gapless boundary modes}

As we saw above, quite generally the Lagrangian (5) is not gauge invariant in the presence of boundaries. For theories where $D_{i}$ are linear in derivatives, this leads to the chiral boundary modes familiar from usual Chern-Simons theories (or stacks thereof). We will now show that gauge invariance requires similar gapless boundary modes in the case at hand.

To be concrete, consider a lattice with a single spatial boundary at $x=0$, and with all fields vanishing as $t \rightarrow \pm \infty$. (With these boundary conditions we may freely integrate by parts in time without incurring additional boundary terms.) From Eq. (6) we deduce that under gauge transformations, the action transforms as

$$
\delta S=-\int_{x=0} \frac{S}{2 \pi}\left(\partial_{u} \partial_{t} \alpha A_{2}-\partial_{t} \partial_{y} \alpha A_{1}-\partial_{u} \partial_{z} \alpha \partial_{y} A_{0}\right) .
$$

To cancel the resulting gauge anomaly, we must add a boundary scalar field $\phi$ to our theory, which transforms as $\phi \rightarrow \phi+\alpha$ under gauge transformations. Upon adding the term

$$
\mathcal{L}_{\mathrm{Bdy}, \phi}=-\frac{s}{2 \pi}\left[A_{2} \partial_{t} \partial_{u} \phi-A_{1} \partial_{t} \partial_{y} \phi-\partial_{u} \partial_{z} \phi \partial_{y} A_{0}\right],
$$

the total action is explicitly gauge invariant.

We now enforce the constraint $B=0$ by writing

$$
A_{1}=\partial_{x} \partial_{u} \alpha, A_{2}=\partial_{y} \partial_{v} \alpha .
$$

Substituting these into the formula (49), and choosing $A_{0}=0$, we obtain the contribution of the gauge fields to boundary action for the scalar field $\alpha$ :

$$
S_{\mathrm{Bdy}, \alpha}=-\int_{x=0} \frac{s}{2 \pi}\left(-\partial_{t} \partial_{u} \alpha \partial_{y} \partial_{z} \alpha\right) .
$$

Note that here we have assumed that we can integrate by parts freely in $y$ and $z$, in order to set

$$
\int_{x=0} \partial_{u} \partial_{t} \alpha \partial_{x} \partial_{y} \alpha-\partial_{y} \partial_{t} \alpha \partial_{x} \partial_{u} \alpha=0
$$

Adding the two contributions to the boundary effective action together, and integrating over the gauge parameter $\alpha$, we obtain the effective action for our boundary scalar field

$$
\mathcal{L}_{\text {Bdy }}=-\frac{s}{2 \pi}\left(\partial_{y} \partial_{z} \phi \partial_{t} \partial_{u} \phi\right) .
$$

To understand what Eq. (54) means for the boundary, let us define

$$
\chi_{i}=\partial_{i} \phi
$$

which is exactly the dipole charge along the $i$ th direction. In terms of the $\chi_{i}$ fields, the boundary action can be expressed as

$$
\mathcal{L}_{\text {Bdy }}=-\frac{s}{2 \pi}\left(\partial_{y} \chi_{z} \partial_{t} \chi_{z}-\partial_{z} \chi_{y} \partial_{t} \chi_{y}\right)
$$

This describes two chiral dipole currents, a $y$-oriented dipole propagating along the $+\hat{z}$ direction and a $z$-oriented dipole propagating along the $-\hat{y}$ direction, at the boundary. However, since the two currents come from the same underlying scalar field $\phi$, the boundary modes are not truly one dimensional in their propagation, and this description must be used with some care.

The discussion above shows that in the presence of a boundary, our higher-rank Chern-Simons theory is incomplete, and extra fields must be added at the boundary to ensure gauge invariance. By definition, the action associated with these fields is also not gauge invariant without the bulk, such that no two-dimensional theory that is invariant under the relevant rank-2 gauge symmetry can exist without the bulk. This is reminiscent of the situation in $(2+1)$-dimensional Chern-Simons theories, where gauge invariance requires chiral boundary modes that are necessarily gapless. There are two important differences, however. First, in the case at hand, rank-2 gauge symmetry in a two-dimensional system requires charge conservation along individual lines, rather than in the system as a whole. Thus, for a boundary at $x=0$, our result implies that no two-dimensional theory in which the total charge is preserved along each $y$ and $z$ line can be described by an action of the form (54). This suggests that if we take this conservation law to be sacred (meaning that we require subsystem symmetry to be preserved at the boundary), then our rank-2 Chern-Simons theory necessarily has gapless surface states. Indeed, a theory of this form was previously used to describe gapless boundary modes protected by subsystem symmetry [32,34,36].

Second, for two-dimensional quantum Hall systems, the boundary modes in the absence of the bulk not only violate charge conservation, they also violate energy conservation. This raises the question of whether there may also be a rank-2 analog of the thermal Hall effect, associated with our surface dipolar flow. As we will see, quantizing our theory on the lattice suggests that this is not the case, though we leave a more thorough discussion of this issue for future work.

\section{DISCRETIZING TO THE LATTICE}

Before we quantize our theory, we first explicitly write a discretization of our theory to the simple cubic lattice. This regularization leads to a quantum theory with a fractonlike ground-state degeneracy, and is closely related to a known fracton lattice model, the Chamon code [14]. We will leave the interesting question of whether other regularizations lead to qualitatively different quantum theories for future investigation.

We begin our discussion by showing how the gauge field content and gauge transformations of our model can arise by gauging a model with an appropriate set of planar U(1) subsystem symmetries. We begin with a model of charged bosons on the cubic lattice, whose Hamiltonian consists of ring-exchange couplings on the three red plaquettes shown in Figs. 7 and 8, perpendicular to the $(0,1,1),(1,0,1)$, and $(1,1,0)$ directions. Specifically,

$$
\begin{aligned}
H= & \sum_{\vec{r}}\left(\phi_{\vec{r}+\hat{y}}^{\dagger} \phi_{\vec{r}+\hat{x}+\hat{y}} \phi_{\vec{r}+\hat{x}+\hat{z}}^{\dagger} \phi_{\vec{r}+\hat{z}}\right. \\
& +\phi_{\vec{r}+\hat{x}}^{\dagger} \phi_{\vec{r}+\hat{y}+\hat{x}} \phi_{\vec{r}+\hat{y}+\hat{z}}^{\dagger} \phi_{\vec{r}+\hat{z}} \\
& \left.+\phi_{\vec{r}+\hat{x}}^{\dagger} \phi_{\vec{r}+\hat{z}+\hat{x}} \phi_{\vec{r}+\hat{z}+\hat{y}}^{\dagger} \phi_{\vec{r}+\hat{y}}\right),
\end{aligned}
$$




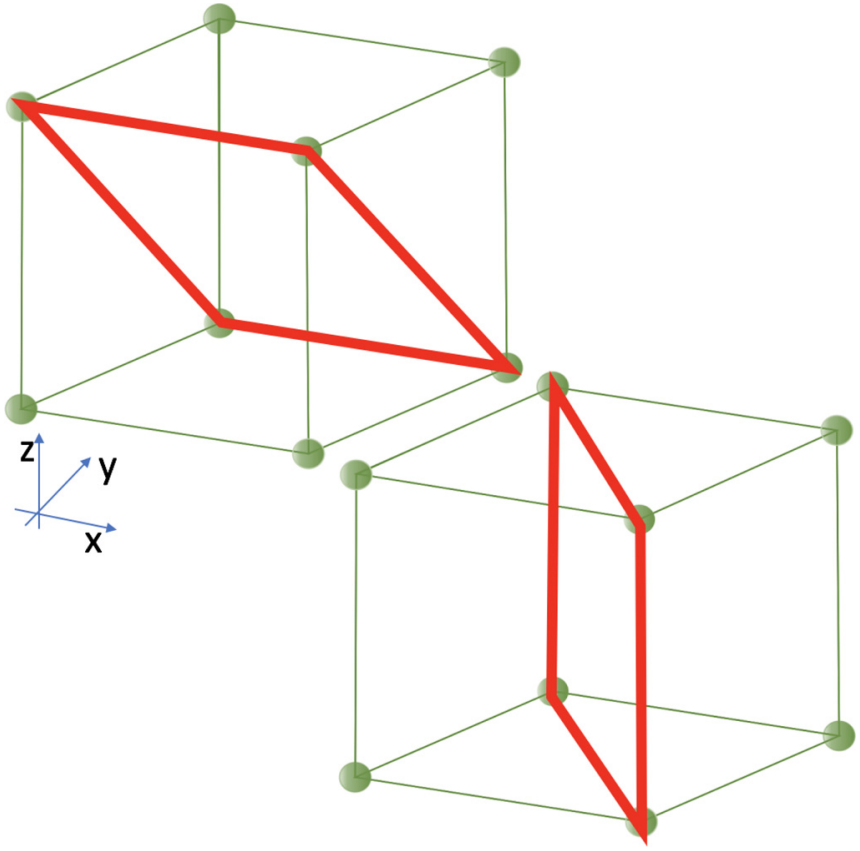

FIG. 7. Plaquettes along which ring-exchange interactions occur in our subsystem-symmetric two-gauge field model.

where $\hat{x}, \hat{y}, \hat{z}$ are the three cubic unit vectors. For convenience, we have set the lattice constant to be 1 , and label sites on the cubic lattice via $\vec{r}=(x, y, z) \in \mathbb{Z}^{3}$.

These ring-exchange interactions preserve the U(1) charge on each $x-y, y-z$, and $x-z$ plane, as well as on the family of lattice planes perpendicular to the $(1,1,1)$ direction. Thus, there are four independent subsystem symmetries.

To obtain our desired higher-rank gauge theory, we place a spatial gauge field $A_{1}, A_{2}$ at the center of each of the two types of plaquette in Fig. 7 , and a timelike gauge field $A_{0}$ at each lattice site. We label these gauge fields via $A_{0,1,2}(\vec{r}, t)$, where $t$ is a continuous-time variable. We use the vector $\vec{r}$ in both cases, even though $A_{1,2}(\vec{r}, t)$ are in fact associated with the dual lattice site at $\vec{r}+\frac{\hat{x}+\hat{y}+\hat{z}}{2}$.

We follow the prescription of Refs. [12,13] to obtain the minimal coupling between these plaquette gauge fields and

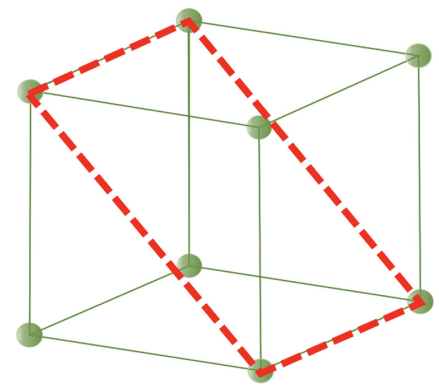

FIG. 8. The product of the two ring-exchange processes shown in Fig. 7 gives a ring-exchange process along a plaquette perpendicular to the $(1,1,0)$ direction, times boson number operators at the remaining corners of the cube. matter. On a plaquette perpendicular to $(0,1,1)$, this gives

$$
\phi_{\vec{r}+\hat{y}}^{\dagger} \phi_{\vec{r}+\hat{x}+\hat{y}} \phi_{\vec{r}+\hat{x}+\hat{z}}^{\dagger} \phi_{\vec{r}+\hat{z}} e^{i A_{1}(\vec{r})} .
$$

For plaquettes normal to $(1,0,1)$ the coupling is analogous, with $A_{1}$ replaced by $A_{2}$. However, because the product of the two ring-exchange terms in Fig. 7 gives rise to a ringexchange process of the type shown in Fig. 8 (times some charge-neutral boson-number operators), the gauge connection on plaquettes perpendicular to the $(1,1,0)$ direction is just $\left(A_{1}+A_{2}\right)$. Thus, our model has only two independent gauge fields on the cubic lattice.

Let us define the forward difference operator in the $x$ direction

$$
d_{x} f(\vec{r}, t)=f(\vec{r}+\hat{x}, t)-f(\vec{r}, t),
$$

and similarly for $d_{y}$ and $d_{z}$. We also define the backward difference operator

$$
\hat{d}_{x} f(\vec{r}, t)=f(\vec{r}, t)-f(\vec{r}-\hat{x}, t),
$$

and similarly $\hat{d}_{y}$ and $\hat{d}_{z}$. Now, we may define the discretized version of our differential operators

$$
\begin{aligned}
& D_{1}=d_{x}\left(d_{y}-d_{z}\right), \\
& D_{2}=d_{y}\left(d_{z}-d_{x}\right)
\end{aligned}
$$

and also $\hat{D}_{1}$ and $\hat{D}_{2}$, with backward difference operators instead. Under a U(1) gauge transformations that takes $\phi_{\vec{r}} \rightarrow$ $e^{i \alpha_{\vec{r}, t}} \phi_{\vec{r}}$, the gauge fields transform as

$$
\begin{aligned}
& A_{0}(\vec{r}, t) \rightarrow A_{0}(\vec{r}, t)+\partial_{t} \alpha(\vec{r}, t), \\
& A_{i}(\vec{r}, t) \rightarrow A_{i}(\vec{r}, t)+D_{i} \alpha(\vec{r}, t) .
\end{aligned}
$$

In the continuum limit, this yields the gauge transformations discussed in Sec. III up to an overall rescaling of the gauge field:

$$
A_{1} \rightarrow A_{1}+\sqrt{2} \partial_{x} \partial_{u} \alpha, \quad A_{2} \rightarrow A_{2}+\sqrt{2} \partial_{y} \partial_{v} \alpha .
$$

We note that the generalized theories described in Sec. III are also naturally described by a model of the type described here, albeit on a distorted cubic lattice, in which the $x-u$ plane is deformed into the $[x+(\alpha-\beta) l]-u$ plane, and similarly for the other directions.

The gauge-invariant electric fields are defined in the same way as before, using these discretized difference operators:

$$
E_{i}(\vec{r}, t)=\partial_{t} A_{i}(\vec{r}, t)-D_{i} A_{0}(\vec{r}, t) .
$$

Each electric field is associated with a cube in the cubic lattice. The magnetic field should be defined with backward difference operators

$$
B(\vec{r}, t)=\hat{D}_{2} A_{1}(\vec{r}, t)-\hat{D}_{1} A_{2}(\vec{r}, t),
$$

hence, each magnetic field is associated with a site, as shown in Fig. 9. One can verify that the $B$ field is gauge invariant by noticing that $\hat{D}_{i}=S_{-x} S_{-y} S_{-z} D_{i}$, where $S_{-x}$ is a shift operator in the $-x$ direction, $S_{-x} f(\vec{r}, t)=f(\vec{r}-\hat{x}, t)$. Then, the variation of $B$ under a gauge transformation is $\delta B=$ $S_{-x} S_{-y} S_{-z}\left(D_{2} D_{1}-D_{1} D_{2}\right) \alpha(\vec{r}, t)=0$. 


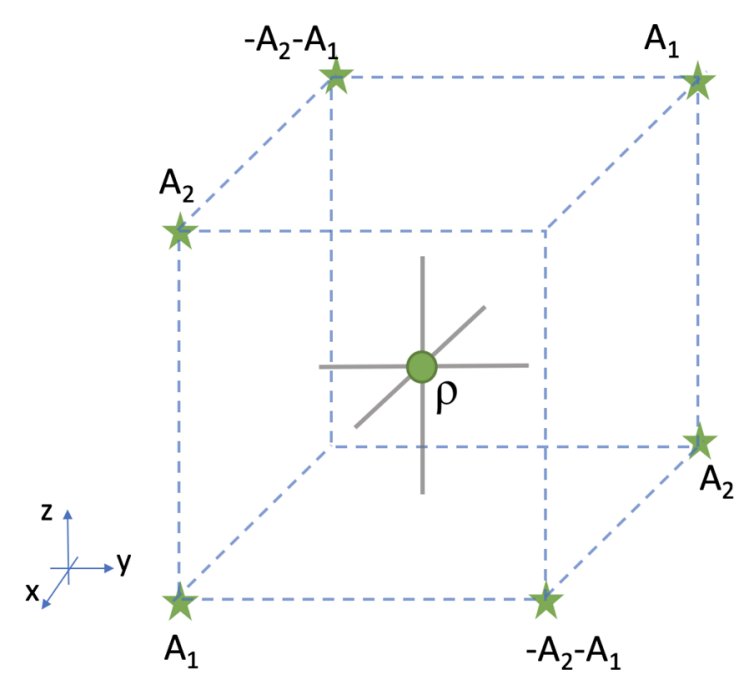

FIG. 9. The Chern-Simons constraint on the cubic lattice. The magnetic field at a given lattice site (green dot at the center of the cube) is given by the linear combination of gauge fields $A_{1}, A_{2}$ at six of the surrounding dual lattice sites (star). The Chern-Simons constraint sets this magnetic field equal to the charge $(\rho)$ on this lattice site.

In the absence of matter sources, the lattice fractonic Chern-Simons action then takes the form

$$
\begin{aligned}
S_{\mathrm{CS}}= & \frac{s}{4 \pi} \int d t \sum_{\vec{r}}\left[A_{1}(\vec{r}, t) E_{2}(\vec{r}, t)\right. \\
& \left.-A_{2}(\vec{r}, t) E_{1}(\vec{r}, t)+A_{0}(\vec{r}, t) B(\vec{r}, t)\right]
\end{aligned}
$$

which one can verify is gauge invariant in the absence of a boundary. For this, one must use a summation by parts, which for our difference operators simply amounts to the identity

$$
\sum_{\vec{r}} f(\vec{r}, t) D_{i} g(\vec{r}, t)=\sum_{\vec{r}}\left(\hat{D}_{i} f(\vec{r}, t)\right) g(\vec{r}, t)
$$

up to boundary terms.

Notice that our theory does not run into the subtle issues associated with discretizing and quantizing the regular 2D Chern-Simons theory (see, for example, Ref. [59]). These subtle issues arise when, for example, canonically conjugate variables do not live on the same location (in 2D CS theory, $A_{1}$ lives on the $x$ links, while $A_{2}$ lives on the $y$ links), or when there are multiple natural choices to be made for the charge-vortex binding (a one-to-one correspondence between plaquettes and vertices is required for the discretized 2D CS theory [60]). Our model sidesteps these issues, as the conjugate variables $A_{1}$ and $A_{2}$ are both located at the center of cubes, and both the $B$ field and charges are located on the vertices (see Fig. 9). Consequently, the lattice discretization does not attribute any subtlety when quantizing the fractonic Chern-Simons term.

\section{A. Gauge-invariant ribbon operators on the lattice}

It is worth briefly discussing how the gauge-invariant line and ribbon operators are manifest on the lattice. To do this, we reintroduce the lattice constant, and imagine that the lattice gauge field $A_{1}^{\text {latt }}(\vec{r}, t)$ is related to a continuum gauge field
$A_{1}^{\text {cont }}(\vec{x}, t)$ by integrating over the associated plaquette:

$$
A_{1}^{\text {latt }}(\vec{r}, t)=\int_{0}^{a} d x \int_{0}^{\sqrt{2} a} d u A_{1}^{\text {cont }}(\vec{r}+x \hat{x}+u \hat{u}, t) .
$$

Note that in the continuum limit, if we assume that $\tilde{A}_{1}$ is smooth, this gives

$$
A_{1}^{\text {latt }}(\vec{r}, t)=\sqrt{2} a^{2} A_{1}^{\text {cont }}(\vec{r}, t)
$$

explaining the relative factor of $\sqrt{2}$ in Eq. (65). The factor of $a^{2}$ gives the expected relationship between the dimensionless lattice gauge field, and our continuum gauge field with dimensions of $1 /$ length $^{2}$.

Since the lattice gauge field is dimensionless, the dimensionful line integrals do not have a lattice analog. However, the dimensionless ribbon operators do since

$$
\int_{x_{0}}^{x_{0}+l a} d x \int_{u_{0}}^{u_{0}+\sqrt{2} a} d u A_{1}^{\mathrm{cont}}(\vec{x}, t)=\sum_{n=0}^{l} A_{1}^{\text {latt }}\left(\vec{r}_{0}+n a \hat{x}, t\right)
$$

and similarly for other directions. In other words, in our lattice theory a ribbon corresponds to a line of plaquettes, with the dipole scale $a$ set by the lattice constant. We will henceforth refer to these operators as lattice Wilson ribbons, or simply Wilson ribbons in contexts where the lattice is understood.

Cage nets on the lattice are constructed from the ribbon operators, exactly as described in the continuum case in Sec. III B. It is straightforward to show that these lattice cage-net operators are fixed by the value of the magnetic field they enclose, and hence that constraint $B=0$ completely fixes these.

\section{QUANTIZING FRACTONIC LATTICE CHERN-SIMONS THEORY}

We now discuss quantization of the fractonic ChernSimons theory, using the lattice regularization introduced in Sec. V. Following Ref. [61], we will quantize within the constrained subspace, meaning that we will first restrict ourselves to configurations where the magnetic field $B(\vec{r}, t)$ defined in Eq. (67) vanishes everywhere. The remaining gauge-invariant operators are the gauge-invariant ribbon operators, and our focus will be on quantizing these in our lattice theory, bearing in mind that not all of them are independent in the constrained Hilbert space.

\section{A. Is the Chern-Simons coefficient quantized?}

Before quantizing the theory, it is useful to ask whether, if the gauge parameter $\alpha \equiv \alpha+2 \pi$ is compact, the Chern-Simons coefficient is quantized. Recall that in ordinary compact U(1) Chern-Simons theory, such quantization is necessary to ensure that large gauge transformations (for example, those that thread a flux of $2 \pi$ through one of the noncontractible curves on the torus) do not actually affect the partition function.

To study this question in more detail, let us consider a gauge transformation of the form

$$
\alpha(x, y, z)= \begin{cases}2 \pi, & x>x_{0}, z>z_{0} \\ 0, & \text { otherwise }\end{cases}
$$




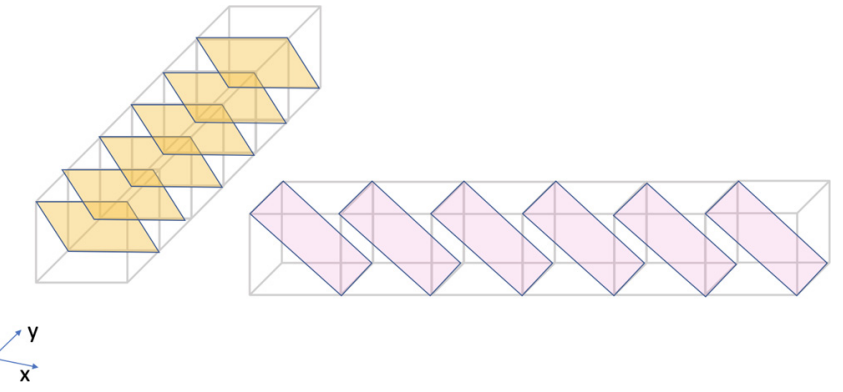

FIG. 10. Large gauge transformations on the lattice consist of changing $A_{1}$ along a line of plaquettes parallel to the $y$ (or $u$ ) direction, or $A_{2}$ along a line of plaquettes parallel to the $x$ (or $v$ ) direction, as shown.

Note that this gauge transformation is allowed if $\alpha \equiv \alpha+2 \pi$; if not it would be incompatible with our choice of periodic boundary conditions. This gauge transformation takes

$$
A_{1}(x, y, z) \rightarrow A_{1}(x, y, z)-2 \pi \delta_{x, x_{0}} \delta_{z, z_{0}},
$$

where $\delta_{x, x_{0}}$ are Kroenecker $\delta$ functions. As explained above, the constraint ensures that $A_{1}$ must be independent of the remaining coordinate $y$.

In this configuration the gauge field $A_{1}$ vanishes everywhere except along a line of plaquettes (see Fig. 10), where it has the value $2 \pi$. The Wilson operators are

$$
\begin{aligned}
\sum_{n=0}^{N_{x}} A_{1}(\vec{r}+n a \hat{x}, t) & =-2 \pi \delta_{z_{0}, r_{3}}, \\
\sum_{n=0}^{N_{u}} A_{1}(\vec{r}+n a(\hat{y}-\hat{z}), t) & =-2 \pi \delta_{x_{0}, r_{1}}
\end{aligned}
$$

indicating that a dipole that encircles the torus along any one of these lines acquires a net phase change of $2 \pi$. Similar results hold for ribbons along the $z$ and $w$ directions, which also involve $A_{1}$. If we take $\alpha$ to be compact, then this phase of $2 \pi$ should not affect the physics at all, and the configuration in Eq. (74) corresponds to a holonomy of our rank-2 gauge field.

Let us now consider the effect of this gauge transformation on the Chern-Simons action. To avoid complications due to boundaries of the manifold in time, we consider periodic boundary conditions in time and space. (Here, as for usual rank-1 Chern-Simons theories, this choice is important since open boundaries require additional fields to preserve gauge invariance.) The net change in our Chern-Simons action is

$$
\begin{aligned}
\mathcal{S}_{\mathrm{CS}} & \rightarrow S_{\mathrm{CS}}-\frac{s}{2 \pi} \sum_{\vec{r}} \int\left(2 \pi \delta_{x, x_{0}} \delta_{z, z_{0}} E_{2}\right) d t \\
& =-s \sum_{y} \int E_{2}\left(x_{0}, y, z_{0}, t\right) d t .
\end{aligned}
$$

Note that to obtain the factor of 2 here comes from integrating by parts in time, which does not induce boundary terms with our choice of periodic boundary conditions.

To complete our analysis, we must understand the quantization of $\sum_{y} \int d t E_{2}$. With periodic boundary conditions in time, we may consider only processes in which the initial and final gauge field configurations are equivalent (up to a gauge transformation). Thus, let $E_{2}$ be the electric field generated by turning on a second holonomy, by taking

$$
A_{2}=\frac{2 \pi t}{\tau} \delta_{y, y_{0}} \delta_{z, z_{0}}
$$

where $\tau$ is the radius of the circular time dimension. Then, $E_{2}$ is constant in time, and

$$
\sum_{y} \int E_{2}\left(x_{0}, y, z_{0}, t\right) d t=2 \pi .
$$

Thus, in order to ensure that the gauge transformation (74) does not change the partition function, the appropriate quantization for our Chern-Simons coefficient is

$$
s \in \mathbb{Z} .
$$

It is worth noting that the above argument must be modified slightly in the continuum theory, where the gauge field has dimensions $1 /$ length $^{2}$, and the Chern-Simons coupling $s$ thus has dimensions of length. In this case, any quantization of the Chern-Simons coupling must depend on some fundamental length scale in the problem, suggesting that the quantized theory requires a fixed ultraviolet cutoff. For this reason, it is natural to quantize the lattice theory, rather than its continuum cousin.

\section{B. Canonical commutation relations}

Having established that for compact U(1) gauge transformations the Chern-Simons couping coefficient is quantized, we are ready to quantize our higher-rank lattice Chern-Simons theory. From the lattice action (68), the canonical commutation relations of the gauge fields $A_{1}, A_{2}$ are

$$
\left[A_{1}(\vec{r}, t), A_{2}\left(\vec{r}^{\prime}, t\right)\right]=i \frac{2 \pi}{s} \delta_{\vec{r}, \vec{r}^{\prime}}
$$

Formally, we wish to work within the constrained subspace where $B=0$, and quantize the remaining gauge-invariant ribbon operators. As discussed in Sec. III A, it is sufficient to consider only ribbon operators involving $A_{1}$ and $A_{2}$, as the values of the remaining ribbon operators involving the linear combination $-A_{1}-A_{2}$ are not independent. Two such ribbon operators necessarily intersect on a single cube, and hence the sums involved share only a single site. Thus, the commutators between intersecting ribbon operators along the major cubic axes are

$$
\begin{gathered}
W_{x} W_{z}=W_{z} W_{x} e^{-i \frac{2 \pi}{s}}, \\
W_{x} W_{y}=W_{y} W_{x} e^{i \frac{2 \pi}{s}}, \\
W_{z} W_{y}=W_{y} W_{z} e^{-i \frac{2 \pi}{s}} .
\end{gathered}
$$

Evidently,

$$
\left[W_{u}, W_{x}\right]=\left[W_{v}, W_{y}\right]=\left[W_{w}, W_{z}\right]=0
$$

while

$$
W_{u} W_{y}=\left(e^{i \frac{2 \pi}{s}}\right)^{b} W_{y} W_{u},
$$

where $b$ counts the number of times that a line along the $u$ direction intersects a line along the $y$ direction. For example, if 
$L_{y} \leqslant L_{z}$ then $b=1$; if $L_{y}=m L_{z}$ with $m \in \mathbb{Z}, b=m$. Similar commutators apply for the remaining directions.

Equation (81), together with the fact that the $W_{i}$ are compact operators, implies that in the quantized theory they are discrete, with a finite set of eigenvalues:

$$
W_{i, j}=e^{2 \pi i l_{i j} / n},
$$

where $n=s / a$, and $l_{i j} \in \mathbb{Z}$.

\section{Ground-state degeneracies}

Since this theory is fully gapped, one telling quantity is the number of ground states. For topological quantum field theories, this number can depend only on the topology of the underlying spatial manifold. In the present case, the groundstate degeneracy is sensitive not only to the topology of the underlying manifold, but also to geometrical factors including the system size and twist angle of the boundary conditions. Here, we will examine this dependence.

The ground states are fully characterized by the eigenvalues of the gauge-invariant line operators in the absence of matter fields. For the case at hand, these are given by the six ribbon operators:

$$
W_{x}, W_{u}, W_{y}, W_{v}, W_{z}, W_{w} .
$$

We begin by considering periodic boundary conditions along the $x, y$, and $z$ directions. In this case, as discussed above, there are $L_{z} / a+L_{y} / a-1$ operators of the type $W_{x}$, and $L_{x} / a+$ $\operatorname{gcd}\left(L_{y} / a, L_{z} / a\right)-2$ additional independent operators of the type $W_{u}$. These can all be simultaneously diagonalized.

Let us first diagonalize all line operators $W_{x}$ running parallel to the $x$ axis. Since every line along $y(z)$ intersects at least one straight line along $x$, we cannot simultaneously diagonalize $W_{x}$ and $W_{y}\left(W_{z}\right)$. Because the lines are straight, however, we may simultaneously diagonalize all $L_{z} / a+L_{y} / a-1$ operators $W_{x}$, and all independent operators of the form $W_{y}\left(x, z_{0}\right)\left(W_{y}\left(x_{0}, z_{0}\right)\right)^{-1}$. Since $\partial_{x} \partial_{z} W_{y}=0$, there are $L_{x} / a-1$ independent operators of this type. (The logic here is that they must be independent of $z$ since the derivative in $x$ cannot vanish.) This set fails to commute with any combinations of line operators parallel to the $z$ axis. Thus, there are a total of $L_{x}+L_{y}+L_{z}-2$ simultaneously diagonalizable line operators along the cubic directions.

Next, we consider whether any of the operators along the $u$ direction can be diagonalized simultaneously with this entire set. The operator $W_{u}$ commutes with $W_{x}$, but in general not with $W_{y}$ ribbons with which it intersects. However, one can choose a set of linear combinations $W_{u}\left(x_{0}, y_{0}+z_{0}\right)\left(W_{u}\left(x_{0}, y_{i}+z_{i}\right)\right)^{-1}$ which commute with all $W_{y}\left(x, z_{0}\right)\left(W_{y}\left(x_{0}, z_{0}\right)\right)^{-1}$ operators and hence these $W_{u}$ lines can be simultaneously diagonalized with the full set described above, leading to an additional $L-1$ line operators on an $L \times L \times L$ system.

Finally, we must determine how many eigenvalues each line operator may take. From taking linear combinations of Eq. (81), we see that lines that intersect once change each others' values by $2 \pi / s$, leading to $s$ possible eigenvalues for each ribbon operator in our set. If we choose $L_{x}=L_{y}=L_{z} \equiv$ $L$ (in which case the diagonal ribbons do not contribute to

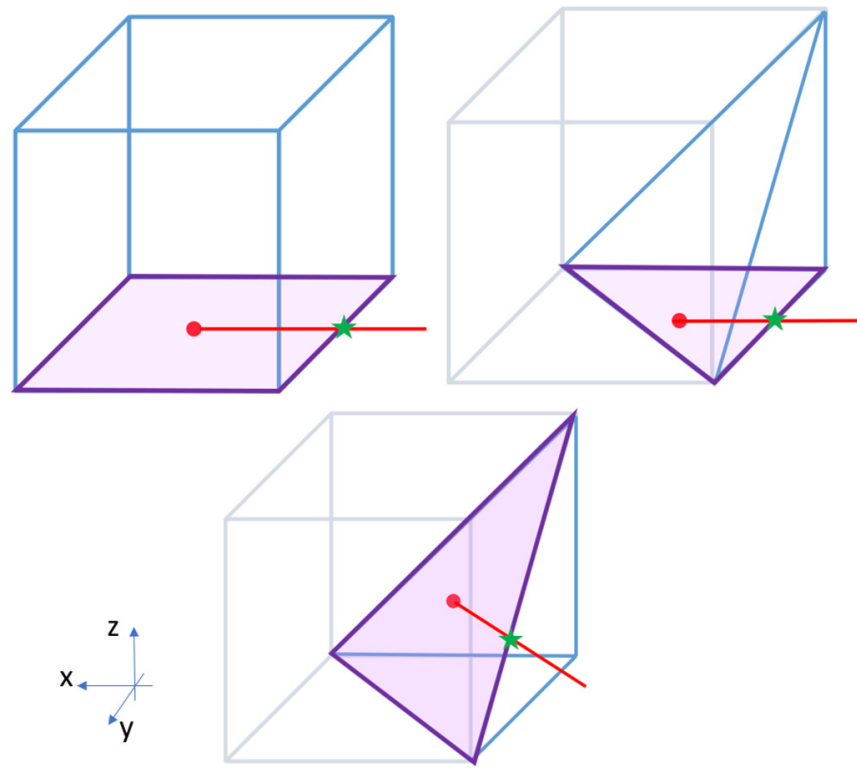

FIG. 11. Schematic of the statistical processes of dipoles in the quantized lattice theory. Red lines represent Wilson ribbons connecting the dipole (represented by a red dot) to a distant antidipole; blue lines represent the ribbon operators of a cage net. Green dots indicate where the dipole and cage-net ribbons cross.

the ground-state degeneracy), we obtain a total degeneracy of $(s)^{4 L / a-3}$ states.

\section{Statistical interactions}

From the commutation relations between the operators $W_{i}$, it is straightforward to infer the quasiparticle statistics. In general, statistical interactions between particles with onedimensional motion (lineons) can be nontrivial only if both particles move in the same plane, such that their world lines intersect. In our model, not all intersecting line operators fail to commute, meaning that some pairs of lineon excitations have trivial mutual statistics. An example are the excitations that travel along the $x$ direction, and those traveling along the $y-z$ direction, both of which are associated with integrals of the gauge field $A_{1}$.

World lines that intersect and fail to commute, such as $W_{x}$ and $W_{y}$, imply that the dipoles have "lineon mutual statistics." Following Refs. [55,57,62], we define these statistics by comparing two processes. In process (a), we first place a dipole at the origin, and then create a dipole-antidipole pair and move the antidipole around in a plane surrounding the origin. As discussed above, any turns in the antidipole's trajectory create other (anti)dipoles; hence, to return the system to its ground state these other dipoles must also be brought together and annihilated; the entire process is represented by a cage net, as shown in Fig. 11. In process (b) we first create, move, and reannihilate the other dipoles to create the cage net, and then (after all of these excitations have vanished) we bring our dipole to the origin. Evidently, the restricted mobility of our dipoles constrains both the planes in which they can encircle each other, and the shapes of the corresponding cage nets.

The braiding phase is determined by the phase difference between processes (a) and (b), which results from the 
commutator between two intersecting ribbon operators, as shown in Fig. 11. For example, if the ribbon ending on the dipole runs along the $\hat{x}$ direction, and the cage net has a surface in the $x-y$ plane, we obtain

$$
M_{a b}=\frac{W_{x} W_{y}}{W_{y} W_{x}}=e^{i 2 \pi / s} .
$$

Similarly, as described in Refs. [34,57], the available cagenet moves can be used to define a type of self-statistics for the lineons. Note that though some aspects of our theory, such as the ground-state degeneracy, are explicitly cutoff dependent, these statistical interactions are scale invariant, depending only on the pattern of crossings between the cage frame and the Wilson ribbon associated with our dipole.

\section{QUANTIZING AND CONSTRAINING: FRACTONIC CHERN-SIMONS THEORY AND THE CHAMON CODE}

Since much of our current understanding of fracton order is based on studying commuting projector lattice Hamiltonians, we now turn to the question of what lattice model of this type could potentially be described by our Chern-Simons theory. To do this, we will consider first quantizing the lattice theory in Sec. V, and then imposing the constraints. We will see how at the second step a mass gap for matter fields results in a Hamiltonian that can be viewed as a $\mathbb{Z}_{s}$ generalization of the Chamon code [14].

We begin with the commutation relations

$$
\left[A_{1}(\vec{r}, t), A_{2}\left(\vec{r}^{\prime}, t\right)\right]=i \frac{2 \pi}{s} \delta_{\vec{r}, \vec{r}^{\prime}} .
$$

Recall that here $\vec{r}, \vec{r}^{\prime}$ refer to sites on the dual cubic lattice, and that gauge fields on different dual lattice sites commute. If $A_{1}$ is compact, then this commutation relation implies that $A_{2}$ is quantized in units of $2 \pi / s$; similarly, if $A_{2}$ is compact, then $A_{1}$ is quantized. Thus, if the gauge fields are compact, our quantized theory is described by an $s$-state spin on each dual lattice site, with

$$
e^{i A_{1}}=U, \quad e^{i A_{2}}=V,
$$

where $U, V$ are $s$-state clock matrices, given by

$$
\begin{aligned}
U_{m n} & =\delta_{m n} e^{2 \pi i n / s}, \\
V_{m n} & \left.=\delta_{m,(n+1} \bmod s\right)
\end{aligned}
$$

such that $U V=e^{2 \pi i / s} V U$.

Next, we must impose the constraint $B=\rho$ at the lattice level. Recall that the lattice magnetic field at site $\vec{r}$ on the direct lattice is given by $B(\vec{r}, t)=\hat{D}_{2} A_{1}(\vec{r}, t)-\hat{D}_{1} A_{2}(\vec{r}, t)$, where the combination of gauge fields is shown in Fig. 9. We have

$$
\begin{aligned}
e^{i B(\vec{r}, t)}= & e^{-i\left[A_{1}(\vec{r}-\hat{x}-\hat{y}, t)+A_{2}(\vec{r}-\hat{x}-\hat{y}, t)\right]} e^{i A_{1}(\vec{r}-\hat{x}, t)} e^{i A_{1}(\vec{r}-\hat{y}-\hat{z}, t)} \\
& \times e^{i A_{2}(\vec{r}-\hat{y}, t)} e^{i A_{2}(\vec{r}-\hat{x}-\hat{z}, t)} e^{\left.-i\left[A_{1}(\vec{r}-\hat{z}, t)\right)+A_{2}(\vec{r}-\hat{z}, t)\right]},
\end{aligned}
$$

where we have used the fact that gauge fields on different sites commute.

In terms of the spin matrices identified in Eq. (88), this product can be expressed as

$$
e^{i B(\vec{r}, t)}=U_{\vec{r}-\hat{x}-\hat{y}}^{\dagger} V_{\vec{r}-\hat{x}-\hat{y}}^{\dagger} U_{\vec{r}-\hat{x}} U_{\vec{r}-\hat{y}-\hat{z}} V_{\vec{r}-\hat{y}} V_{\vec{r}-\hat{x}-\hat{z}} V_{\vec{r}-\hat{z}}^{\dagger} U_{\vec{r}-\hat{z}}^{\dagger},
$$

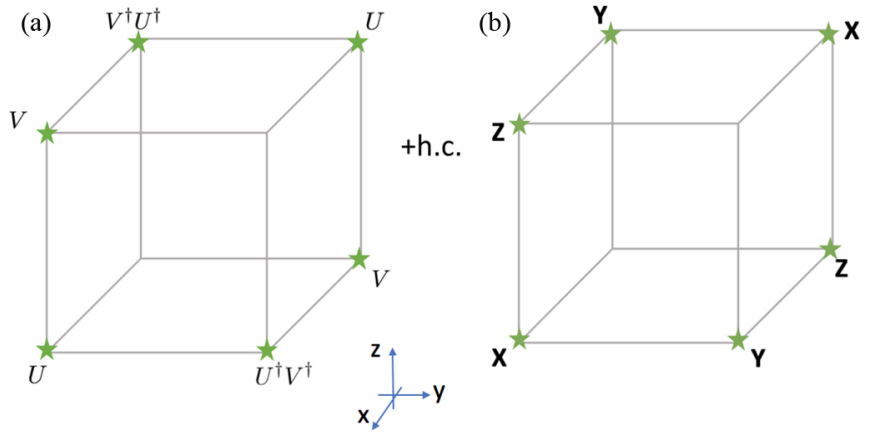

FIG. 12. (a) The magnetic field operator in our quantized (but unconstrained) theory. Adding a mass term for matter fields and imposing the Chern-Simons constraint $B=\rho$ leads to a Hamiltonian that can be expressed as the product of the six operators shown, plus its Hermitian conjugate. (b) For $s=2$, this construction gives the tilted Chamon code.

where we have used

$$
e^{-i\left[A_{1}(\vec{r}, t)+A_{2}(\vec{r}, t)\right]}=e^{-i A_{1}(\vec{r}, t)} e^{-i A_{2}(\vec{r}, t)} e^{-i \pi / s} .
$$

The result is a product of six spin operators at the corners of the cube, as shown in Fig. 12.

The lattice Hamiltonian corresponding to our pure ChernSimons theory is then

$$
H=-\frac{1}{2} \sum_{\vec{r}}\left(e^{i B(\vec{r}, t)}+e^{-i B(\vec{r}, t)}\right) .
$$

Clearly, the ground states of this model obey $B(\vec{r}, t) \equiv 0$, corresponding to the manifold of states of the Chern-Simons theory in the absence of sources. The excited states can be understood as the result of introducing gapped, nondynamical matter sources on the sites of our lattice. After imposing the constraint $B(\vec{r}, t)=\frac{2 \pi}{s} \rho(\vec{r}, t)$, the mass gap for these nondynamical sources leads to a Hamiltonian of the form (93).

An interesting example is $s=2$. In this case, we have $\sigma^{x}=U, \sigma^{z}=V$, and $U V=-i \sigma^{y}$, which obeys the required algebra $U V=-V U$. In this case, our Hamiltonian (93) becomes

$$
H=-\sum_{\vec{r}} \sigma_{\vec{r}-\hat{x}}^{x} \sigma_{\vec{r}-\hat{y}-\hat{z}}^{x} \sigma_{\vec{r}-\hat{x}-\hat{y}}^{y} \sigma_{\vec{r}-\hat{z}}^{y} \sigma_{\vec{r}-\hat{x}-\hat{z}}^{z} \sigma_{\vec{r}-\hat{y}}^{z} .
$$

This is exactly the Chamon code [14] with a tilted geometry [63]. In retrospect, this correspondence is quite natural: the Chamon code has six types of lineon operators, along the three cubic axes and three diagonal directions, each of which creates a distinct lineon-type excitation free to move only along that linear direction. The mobility of these excitations, together with the Wilson-line algebra of the Chamon code, coincide with our Chern-Simons gauge theory at $s=2$. By counting the number of independent stabilizers in Eq. (94), one can also see directly that the ground-state degeneracy of the tilted Chamon code is $2^{4 L-3}$ on an $L \times L \times L$ lattice with periodic boundary conditions, exactly as predicted for our Chern-Simons theory.

The original Chamon code on the fcc lattice, which has full cubic symmetry, can be obtained by quantizing the generalized fracton Chern-Simons theory described in Sec. III C, 


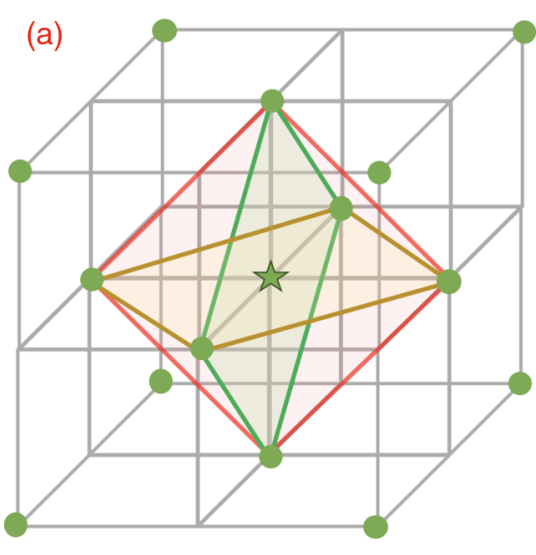

(b)
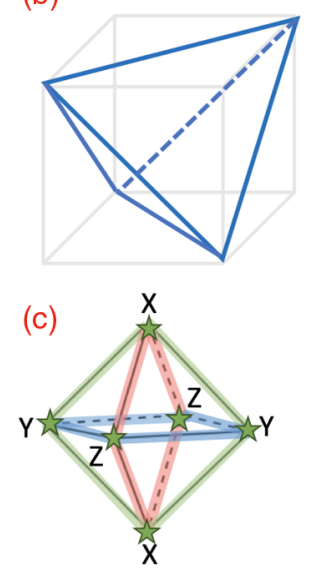

FIG. 13. Geometry of the $\alpha=2, \beta=1$ theory corresponding to the Chamon code on the fcc lattice. (a) The gauge fields $A_{1}, A_{2}$ live on the dual lattice (star) at the centers of octahedral plaquettes. The matter fields live at sites (dot) of the direct lattice. $e^{i A_{1}}$ is coupled to the four charges at the corners of the red plaquette; $e^{i A_{2}}$ is coupled to the four charges at the corners of the green plaquette. (b) The cage-net configuration. (c) The magnetic field operator for $s=2$ (i.e., for the Chamon code). Sites shown here represent sites on the dual lattice.

with $s=2$. Specifically, by taking differential operators of the form (42), with $\alpha=2, \beta=1$, we obtain

$$
\begin{aligned}
& D_{1}=\left(\partial_{y}+\partial_{z}\right)\left(\partial_{y}-\partial_{z}\right)=\left(\partial_{y}^{2}-\partial_{z}^{2}\right), \\
& D_{2}=\left(\partial_{z}+\partial_{x}\right)\left(\partial_{z}-\partial_{x}\right)=\left(\partial_{z}^{2}-\partial_{x}^{2}\right) .
\end{aligned}
$$

To get a lattice model in agreement with Chamon's code, we define the discretized differential operators as

$$
\begin{aligned}
& D_{1}=\left(d_{y} \hat{d}_{y}-d_{z} \hat{d}_{z}\right), \\
& D_{2}=\left(d_{z} \hat{d}_{z}-d_{x} \hat{d}_{x}\right),
\end{aligned}
$$

where $d_{i}$ and $\hat{d}_{i}$ are forward and backward difference operators on the cubic lattice (59). As Chamon's code is defined on the fcc lattice, we place gauge fields $A_{1}(\vec{r}, t)$ and $A_{2}(\vec{r}, t)$ on the $a$ sublattice of the simple cubic lattice, while the matter fields [and $\alpha(\vec{r}, t)$ ] live on the $b$ sublattice. The $a$ sublattice on which $A_{1}$ and $A_{2}$ live therefore forms an fcc lattice. Consequently, the $E$ and $B$ fields of this lattice model live on the $a$ and $b$ sublattice, respectively. The resulting gauge theory has charge conservation on (1 1 1 1), ( $11-1),(-111),\left(\begin{array}{lll}1 & 1 & 1\end{array}\right)$ planes. The gauge-invariant cage nets form symmetric tetraheda, whose edges lie in the $x \pm y, y \pm z, x \pm z$ directions, as shown in Fig. 13. The corresponding lattice gauge theory can be obtained by gauging a plaquette ring-exchange model with ring-exchange terms on the three plaquettes of the octahedron shown in Fig. 13. These ring-exchange processes conserve charge on (111), (1 1-1), (-1 11), (1-11) planes. After gauging the resulting $\mathrm{U}(1)$ subsystem symmetries following the method described in Sec. V, we obtain two gauge fields $A_{1}, A_{2}$ at the center of each octahedron, with gauge transformations,

$$
\begin{aligned}
& A_{1} \rightarrow A_{1}+\left(d_{y} \hat{d}_{y}-d_{z} \hat{d}_{z}\right) \alpha, \\
& A_{2} \rightarrow A_{2}+\left(d_{z} \hat{d}_{z}-d_{x} \hat{d}_{x}\right) \alpha .
\end{aligned}
$$

The resultant gauge theory contains lineon excitations extended along the $\hat{i} \pm \hat{j}$ directions, whose end points carry dipoles oriented in $\hat{i} \mp \hat{j}$. With this definition of $D_{1}, D_{2}$, we obtain a lattice Hamiltonian

$$
\begin{aligned}
& H=-\sum_{\vec{r}} \cos (B(\vec{r}, t)) \\
& =-\sum_{\vec{r} \in b} \sigma_{\vec{r}+\hat{x}}^{x} \sigma_{\vec{r}-\hat{x}}^{x} \sigma_{\vec{r}+\hat{y}}^{y} \sigma_{\vec{r}-\hat{y}}^{y} \sigma_{\vec{r}+\hat{z}}^{z} \sigma_{\vec{r}-\hat{z}}^{z}
\end{aligned}
$$

which, as above, can be viewed as resulting from introducing massive nondynamical matter sources, and imposing the Chern-Simons constraint $B(\vec{r}, t)=\rho(\vec{r}, t)$. Here, $\vec{r} \in b$ sums over $b$ sublattice sites denoted by the dots in Fig. 13. This exactly reproduces the Hamiltonian and one-dimensional excitations of the Chamon code on fcc lattice formed by the $a$ sublattice of our simple cubic lattice.

One can also define lattice Chern-Simons theories with $s>2$ in this geometry, following the procedure outlined above. Note, however, that despite the seeming cubic symmetry of this model, for $s>2$ our lattice Chern-Simons theories do not have cubic symmetry. This is because for $s>2$ the operator $B$, and therefore the Chern-Simons action, is odd under $C_{4}^{i}$ rotations. Interestingly, however, in the absence of matter we have $B=0$, and the resulting ground states of the lattice model are invariant under full cubic symmetry. ${ }^{4}$

\section{GAPLESS HIGHER-RANK CHERN-SIMONS THEORIES WITH THREE GAUGE FIELDS IN THREE DIMENSIONS}

The theories described thus far are tensor gauge theories in the sense that the gauge transformations are quadratic in derivatives; however, they do not correspond to any higherrank gauge theories discussed in the literature so far. This is because, if our charge is a scalar, then our Chern-Simons theory is gapped only if we have at most two gauge fields. In three-dimensional symmetric tensor gauge theories, the natural number of gauge fields is either 3 (if $A_{i j}$ is an offdiagonal symmetric tensor) or 6 (for a general symmetric tensor). To make contact with these theories, here we will briefly describe the fate of Chern-Simons theory of the symmetric, off-diagonal tensor gauge theory. The main interesting feature of this theory is that, unlike the pure Maxwell theory [56], Maxwell-Chern-Simons theory of off-diagonal symmetric tensor gauge fields in three dimensions is deconfined.

We consider a symmetric off-diagonal tensor gauge theory with the gauge fields $A_{x y}, A_{x z}$, and $A_{y z}$. This off-diagonal tensor structure is not invariant under continuous rotations in three dimensions, but rather only under the symmetries of a cubic lattice. The gauge transformations of this theory are $[26,29,34,45]$

$$
A_{i j} \rightarrow A_{i j}+\partial_{i} \partial_{j} \alpha, A_{0} \rightarrow A_{0}+\partial_{t} \alpha .
$$

Note that our gauge parameter $\alpha$ is a scalar, indicating that this is a scalar charge theory, in the language of

\footnotetext{
${ }^{4}$ For $s=2$, the $\pm \pi$ fluxes are equivalent, so in this case the full theory has cubic symmetry.
} 
Refs. [26,29,34,45]. The gauge-invariant electric and magnetic fields are

$$
\begin{aligned}
E_{i j} & =\partial_{t} A_{i j}-\partial_{i} \partial_{j} A_{0}, \\
B_{x} & =\partial_{y} A_{x z}-\partial_{z} A_{x y}, \\
B_{y} & =\partial_{z} A_{x y}-\partial_{x} A_{y z}, \\
B_{z} & =\partial_{x} A_{y z}-\partial_{y} A_{x z} .
\end{aligned}
$$

The magnetic fields satisfy $\sum_{i} B_{i}=0$, such that there are only two independent field components. The gauge-invariant ribbon operators have the form

$$
\oint_{C(x, y)} \sum A_{i z} \mathbf{d l}_{i}
$$

for $C(x, y)$ any closed curve in the $x, y$ plane, and similarly in the other directions. Note that unlike the theories discussed in previous sections of this paper, coupling this theory to matter leads to dipolar excitations (planeons) that are mobile in twodimensional planes.

In this theory, it is not possible to write a Chern-Simons action imposing the constraint $B=\rho$ since our charge $\rho$ is a scalar, but the magnetic field $B$ is not. As $\sum_{i} B_{i}=0$, the lowest-order constraint that does not violate threefold rotational symmetry about the $(1,1,1)$ direction is therefore

$$
\frac{s}{2 \pi} \partial_{i} B_{i}=\rho .
$$

To enforce this constraint, we choose the Chern-Simons action to be

$$
\mathcal{L}_{\mathrm{CS}}=\frac{s}{4 \pi} \epsilon^{i j k}\left(A_{j k} \partial_{t} A_{i j}+2 \partial_{j} A_{0} \partial_{i} A_{j k}\right)-A_{0} \rho-A_{i j} J_{i j} .
$$

Because constraint (103) is not sufficient to fully fix the magnetic field, the pure Chern-Simons theory is unstable, and contains an extensive number of ground states in any geometry. Instead, we consider a Maxwell-Chern-Simons theory, of the form

$$
\mathcal{L}=-\frac{1}{2 g^{2}}\left(\sum_{i j} E_{i j}^{2}+\sum_{i} B_{i}^{2}\right)+\mathcal{L}_{\mathrm{CS}} .
$$

In the absence of sources, the equations of motion are

$$
\begin{aligned}
\sum_{i j} \partial_{i} \partial_{j} E_{i j}+\frac{s}{2 \pi} \sum_{i} \partial_{i} B_{i} & =0, \\
\partial_{t} E_{x y}+\frac{s}{2 \pi}\left(E_{y z}-E_{x z}\right) & =\partial_{z}\left(B_{x}-B_{y}\right), \\
\partial_{t} E_{y z}+\frac{s}{2 \pi}\left(E_{x z}-E_{x y}\right) & =\partial_{x}\left(B_{y}-B_{z}\right), \\
\partial_{t} E_{x z}+\frac{s}{2 \pi}\left(E_{x y}-E_{y z}\right) & =\partial_{y}\left(B_{z}-B_{x}\right) .
\end{aligned}
$$

In addition, the analogs of the homogeneous Maxwell equations are

$$
\begin{gathered}
\sum_{i} B_{i}=0, \\
\partial_{z} E_{x y}-\partial_{y} E_{x z}=-\partial_{t} B_{x}, \\
\partial_{y} E_{x z}-\partial_{x} E_{y z}=-\partial_{t} B_{z}, \\
\partial_{x} E_{y z}-\partial_{z} E_{x y}=-\partial_{t} B_{y} .
\end{gathered}
$$

One can solve Eqs. (107) and (106) to reveal two modes, with frequencies

$$
\omega_{ \pm}^{2}=\vec{k}^{2}+\frac{3}{2} s^{2} \pm \sqrt{\Delta_{\vec{k}}^{4}+s^{2}\left(k_{x}+k_{y}+k_{z}\right)^{2}+\frac{9}{4} s^{4}},
$$

$\omega_{-}$is gapless as $\vec{k} \rightarrow 0$, while $\omega_{+}$has a gap proportional to the Chern-Simons coupling $s$. Thus, in the infrared our action (105) describes a symmetric off-diagonal tensor gauge theory with a single propagating gapless mode.

To better understand this gapless fixed point, it is convenient to add off-diagonal couplings in the electric fields; the symmetric combination of these violates no lattice symmetries and is thus allowed. This allows us to consider the action

$$
\begin{aligned}
\mathcal{L}= & \frac{s}{4 \pi}\left[\sqrt{3}\left(-A_{u} \partial_{t} A_{v}+A_{v} \partial_{t} A_{u}\right)-2 A_{0} \partial_{i} B_{i}\right] \\
& +\frac{1}{2 g^{2}}\left(E_{\ell}^{2}+\sum_{i} B_{i}^{2}\right),
\end{aligned}
$$

where

$$
E_{\ell}=\sum_{i j} E_{i j}
$$

We note that this particular choice of Lagrangian has the peculiarity that the massive branch of solutions to MaxwellChern-Simons theory are entirely absent; thus, we do not need to project out any high-energy modes in order to study the long-wavelength theory.

\section{A. Confinement vs Chern-Simons terms}

It is known [56] that in the absence of the Chern-Simons term, the Lagrangian (105) leads to a confining theory for all values of the gauge coupling $g$. We now show that the ChernSimons term prevents confinement, by a mechanism similar to that identified by Ref. [58] in $(2+1)$-dimensional ChernSimons theories.

First, let us review the nature of confinement in the Maxwell theory. Reference [56] showed that in the absence of a Chern-Simons term, if the U(1) gauge field is compact, then $2 \pi$ flux defects will proliferate, confining the theory. These defects correspond to introducing a $2 \pi$ branch plane in the gauge parameter $\alpha$, that emenates from the origin along, for example, the $\hat{x}, \hat{z}$ axes. We define the branch plane by a singularity in the derivative of $\alpha$, as follows:

$$
\partial_{y} \alpha=2 \pi \delta(y) \theta(z) \theta(x) .
$$

From this, we see that $\partial_{x} \partial_{y} \alpha=2 \pi \delta(y) \delta(x) \theta(z)$ is well defined away from $z=0$, and similarly that $\partial_{z} \partial_{y} \alpha=2 \pi \delta(y) \delta(z) \theta(x)$ is well defined away from $x=0 . \partial_{x} \partial_{z} \alpha$ is well defined (and vanishes) away from the origin.

To see that this branch cut introduces magnetic flux at the origin, consider a region $R$ about the origin bounded a curve $C$ in the $x-y$ plane, and stretching from $-l / 2$ to $l / 2$ in the $z$ direction. Assuming that our gauge fields are pure gauge, 
along such a ribbon, we have

$$
\begin{aligned}
\int_{R} B_{z} & =\oint_{C}\left(\int_{-l / 2}^{l / 2} A_{i z} d z\right) d x_{i} \\
& =\oint_{C}\left(\int_{-l / 2}^{l / 2} \partial_{z} \partial_{i} \alpha\right) d x_{i} \\
& =2 \pi \oint_{C} \delta(y) \theta(x) d x_{i}=2 \pi,
\end{aligned}
$$

where the last equality holds because the ribbon crosses the $x$ axis at a single point. For a ribbon that does not enclose the origin, this quantity is 0 . Thus, inserting a branch sheet of this type in $\alpha$ can be viewed as a large gauge transformation, which changes the $z$ and $x$ components of the magnetic flux by $\pm 2 \pi$ at the origin. These are precisely the topological defects that proliferate to drive confinement [56].

Next, we show that such defects cannot proliferate in the presence of a Chern-Simons term. To see this, we first note that the Chern-Simons term is gauge invariant in the bulk only when the homogeneous Maxwell equations (107) are satisfied. Specifically, under a gauge transformation by $\alpha$ the bulk Chern-Simons action changes according to

$$
\delta \mathcal{L}_{\mathrm{CS}}=\frac{s}{2 \pi} \alpha \partial_{i}\left(\epsilon^{i j k} \partial_{j} E_{k i}+\partial_{t} B^{i}\right) .
$$

However, large gauge transformations like the one described above stem from processes in which the homogeneous Maxwell equations are violated. To see this, we integrate the homogeneous Maxwell equations (107) over some spatial region $R$ :

$$
\dot{\Phi}_{i}(R)=\int_{0}^{l} d x_{i} \int_{S} \sum_{j, k} \epsilon^{i j k} \partial_{j} E_{k i}=\int_{0}^{l} d x_{i} \oint_{\partial S} E_{i j}(d l)_{j} .
$$

Here,

$$
\phi_{i}(R)=\int_{R} \phi_{i}(R) d^{3} \mathbf{r}
$$

and we have taken the region $R$ to have length $l$ in the direction parallel to $i$, and span a surface $S$ in the transverse directions. We can see that on a closed manifold, or on an infinite manifold with appropriate boundary conditions, if we take $i=x$ and $S$ to be the entire $y-z$ plane, then the right-hand side must vanish. Thus, processes that change the magnetic flux through any planar region (of width $l$ ) necessarily fail to satisfy the homogeneous Maxwell's equations, and thus are not gauge invariant in the presence of a Chern-Simons term. Thus, exactly as in the case of usual Chern-Simons theories in $2+1$ dimensions, integrating over the gauge parameter in the partition function suppresses these processes, and thus prevents confinement.

An example of a continuum space-time process that inserts a a flux of $2 \pi$ in $B_{x}$ is the monopole like solution

$$
\begin{aligned}
\left(-E_{x z}, E_{x y}, B_{x}\right)= & \frac{1}{2}\left(\frac{y}{\left(y^{2}+z^{2}+t^{2}\right)^{3 / 2}}, \frac{z}{\left(y^{2}+z^{2}+t^{2}\right)^{3 / 2}},\right. \\
& \left.\frac{t}{\left(y^{2}+z^{2}+t^{2}\right)^{3 / 2}}\right) .
\end{aligned}
$$

It is easy to check that with this solution, $\int d y d z B_{x}=$ $2 \pi \theta(t)$, so that the magnetic flux changes by $2 \pi$. On the other hand, the homogeneous Maxwell equation (107) requires that the divergence of the vector defined in Eq. (116) vanish. For our solution this is the case everywhere except at $y=z=t=$ 0 , where it is singular; one can check that this singularity has the form $\partial_{z} E_{x y}-\partial_{y} E_{x z}+\partial_{t} B=\delta((y, z, t)-(0,0,0))$, leading to a gauge-dependent contribution to the Chern-Simons action.

\section{B. Quantized gapless theory}

Finally, let us study the properties of our quantized gapless theory. The canonical commutation relations from our Lagrangian (109) are

$$
\begin{aligned}
& {\left[A_{x y}, \frac{s}{2 \pi}\left(A_{x z}-A_{y z}\right)+\frac{1}{\sqrt{3} g^{2}} E_{\ell}\right]=i,} \\
& {\left[A_{x z}, \frac{s}{2 \pi}\left(A_{y z}-A_{x y}\right)+\frac{1}{\sqrt{3} g^{2}} E_{\ell}\right]=i,} \\
& {\left[A_{y z}, \frac{s}{2 \pi}\left(A_{x y}-A_{x z}\right)+\frac{1}{\sqrt{3} g^{2}} E_{\ell}\right]=i .}
\end{aligned}
$$

In addition, we have $\left[A_{y z}-A_{x z}, E_{\ell}\right]=\left[A_{x y}-A_{x z}, E_{\ell}\right]=0$. It follows that

$$
\frac{1}{\sqrt{3} g^{2}}\left[A_{i j}, E_{\ell}\right]=\frac{i}{3} .
$$

One can use this to determine the remaining commutation relations between the $A_{i j}$ :

$$
\begin{aligned}
& {\left[A_{x y}, A_{x z}\right]=\frac{2 \pi}{3 s} i,} \\
& {\left[A_{x z}, A_{y z}\right]=\frac{2 \pi}{3 s} i,} \\
& {\left[A_{y z}, A_{x y}\right]=\frac{2 \pi}{3 s} i .}
\end{aligned}
$$

In Appendix D, we show that line operators of the form (102) commute with the constraint, and are thus all allowed within the low-energy theory. However, in general these line operators do not preserve the magnetic field or $E_{\ell}$. For example,

$$
\left[\int d x A_{x z}(\mathbf{r}), B_{x}\left(\mathbf{r}^{\prime}\right)\right]=i s \int d x \partial_{y} \delta\left(\mathbf{r}-\mathbf{r}^{\prime}\right)
$$

which is not, in general, 0 . Thus, we conclude that the line operators in general do not commute with the Hamiltonian, and thus do not keep the system in its ground state. To see this explicitly, let $\hat{W}=e^{i \int A_{i j}}$ represent a unitary Wilson ribbon operator. Since $e^{i A_{i j}}$ is a raising operator for $E_{\ell}$ (for any $i, j$ ), the energy difference between a state with and without having acted with the Wilson ribbon is

$$
\begin{aligned}
\left\langle\hat{W}^{\dagger}\right. & \left.\Psi\left|E_{\ell}^{2}\right| \hat{W} \Psi\right\rangle-\left\langle\Psi\left|E_{\ell}^{2}\right| \Psi\right\rangle \\
& =\left\langle\Psi\left|\hat{W}^{\dagger}\left[E_{\ell}^{2}, \hat{W}\right]\right| \Psi\right\rangle \\
& =\left\langle\Psi\left|\hat{W}^{\dagger} E_{\ell} \hat{W}+E_{\ell}\right| \Psi\right\rangle \\
& =\left\langle\Psi\left|\frac{g^{2}}{\sqrt{3}}+2 E_{\ell}\right| \Psi\right\rangle .
\end{aligned}
$$


Thus, the Wilson ribbon creates a line of electric field along the path of the Wilson ribbon, much as occurs in ordinary Maxwell theory. A similar argument shows that a Wilson line also generates magnetic flux. Thus, in the presence of low-energy gapless modes, the Wilson-line operators do not map between different quantum states of the same energy, in spite of the fact that they relate different classical ground-state configurations. Further, as Wilson lines are not associated with large gauge transformations in the quantum theory, $a$ priori we do not expect the Chern-Simons coefficient to be quantized in this case as the gapless gauge fluctuation could modify the Chern-Simons coupling.

In our gapless theory, dipoles (which appear at the end of open ribbon operators) create electric and magnetic fields as they move about, and thus have long-ranged Coulomb-type interactions. However, they also acquire a statistical interaction from the nontrivial commutators of their ribbons. This statistic is between dipoles of the same orientation (which are restricted to move in the same $2 \mathrm{D}$ plane), though there is also a contact interaction (that is not topological in nature) between crossing lines of dipoles with different orientation.

\section{OUTLOOK}

We have investigated how a field-theoretically motivated approach to constructing TQFT-like actions for higher-rank gauge fields in three spatial dimensions leads to a number of insights about the possibilities for fractonic tensor gauge theories. First, we have outlined one general philosophy for writing such terms, consisting of identifying gauge-invariant (in the bulk) actions that impose a constraint binding charge to the higher-rank gauge flux, and discussed its interplay with symmetry. We have described both Chern-Simons-type and BF-like versions of this construction, though our main focus has been on the former.

Second, we have presented a detailed analysis of both the classical and (lattice-regularized) quantum versions of one particular gapped fractonic Chern-Simons theory. Notably, by analyzing the gauge transformations of Wilsontype oeprators, we have seen how even in the absence of a Gauss' law constraint, the structure of gauge transformations restricts charges' mobility, giving an alternate perspective on the paradigm developed by Ref. [31]. We have also identified several ways in which this theory is qualitatively distinct from fracton-inspired field theories in the existing literature, including the presence of time-reversal symmetry-breaking gapless boundary modes in the classical theory, and selfstatistics for charged particles upon quantization. Finally, we have established a strong correspondence between our theory and fracton order, both in terms of its physical properties (such as restricted quasiparticle mobility and the scaling of the ground-state degeneracy with system size), and by establishing a correspondence to a lattice Hamiltonian that can be viewed as a generalized Chamon code. To the best of our knowledge, this $Z_{s}$ generalization of the Chamon code has not previously appeared in the literature.

Third, we have briefly described a scenario that has not previously been considered in the context of higher-rank gauge theories, of a gapless higher-rank Maxwell ChernSimons theory, with both spontaneously broken time-reversal symmetry and long-ranged Coulomb-type interactions. This scenario is interesting in part because it demonstrates how a higher-rank Chern-Simons term can prevent confinement.

Our work raises several interesting questions. First, the observation that our fractonic Chern-Simons theory is gauge invariant only up to a boundary term is surprising in light of the fact that our quantized lattice gauge theory can be mapped exactly onto a commuting projector Hamiltonian. In particular, this suggests that in spite of the chiral nature of the resulting pattern of dipolar current flow on the boundary, our fractonic Chern-Simons theory may not have ungappable chiral boundary states, as are present in $(2+1)$ D quantum Hall systems. We defer a better understanding of how our lattice model preserves gauge invariance at the boundary for future work.

Second, here we have used a lattice regularization in order to quantize our theory. This avoids several issues that arise in the continuum with a compact U(1) theory. Further, a lattice regularization naturally captures the geometrical aspects of fracton theories, such as the dependence of the ground-state degeneracy on system size. Nevertheless, it would be interesting to study other possible regularizations, and understand whether, or in what sense, a truly continuum version of these quantum field theories exists.

Third, it is clear that the construction described here admits several generalizations. First, the higher-rank BF theories described in Sec. II can be constructed for theories whose gauge transformations are not purely second order in derivatives, but more general polynomials in the momenta. This framework can potentially be used to explore field theories with fractonic current conservation laws that result from general subsystem symmetries [49], including type-II fracton theories [10,31]. Second, our construction can be extended to vector-charge theories, or (in the case of BF-like actions) to hybrid theories with both vector and scalar charges. This allows one to contemplate actions that impose a much broader class of constraints. Such generalizations are clearly necessary to capture many known fracton orders, and replicate existing field theories such as Ref. [16]'s description of the X-cube model.

\section{ACKNOWLEDGMENTS}

We are grateful to K. Slagle, C. Xu, X. Chen, A. Gromov, and M. Hermele for helpful discussions. Y.Y. is supported by PCTS Fellowship at Princeton University. F.J.B. is grateful for the financial support of NSF-DMR Grant No. 1352271 and the Sloan Foundation Grant No. FG-2015-65927. Y.Y. and F.J.B. performed part of this work at the Aspen Center for Physics, which is supported by National Science Foundation Grant No. PHY-1607611.

\section{APPENDIX A: ALLOWED DIRECTIONS OF LINE OPERATORS}

To see that the line operators must run along certain directions, we consider an arbitrary linear combination of the two gauge fields

$$
A_{l}=a A_{u}+b A_{v} .
$$


Under gauge transformations,

$$
A_{l} \rightarrow A_{l}+(a+b) \partial_{x} \partial_{y} \alpha-(a-b) \partial_{x} \partial_{z} \alpha-2 b \partial_{y} \partial_{z} \alpha .
$$

In order for a line operator of the form $\int A_{0} d x_{l}$ to be gauge invariant, it must be the case that $A_{l} \rightarrow A_{l}+\left(\sum_{k} a_{k} \partial_{k}\right) \partial_{l} \alpha$; in other words, the gauge transformation must factor. However, since the gauge transformation contains no terms quadratic in derivatives along any of the cubic axes, it must have the form

$$
(a+b) \partial_{x} \partial_{y} \alpha-(a-b) \partial_{x} \partial_{z} \alpha-2 b \partial_{y} \partial_{z} \alpha=\left(c \partial_{x}+d \partial_{y}\right) \partial_{z} \alpha
$$

or similarly with the $x, y$, and $z$ labels permuted on the right-hand side. It is easy to check that the only solutions to this equation have $d=-c$, for which we obtain the linear combinations of gauge fields $A_{u}, A_{u^{*}}$, and $A_{u^{* *}}$ given above. Thus, the six line operators identified in the text exhaust all gauge-invariant line operators in our model.

\section{APPENDIX B: IRREDUCIBLE REPRESENTATIONS OF $C_{3}$}

The rotation group $C_{3}$ in three dimensions has four irreducible representations: two scalar representations $\Gamma_{A_{1}}, \Gamma_{A_{2}}$, and two two-dimensional representations which we will call $\Gamma_{a}, \Gamma_{b}$. These descend from the vector $\left(L_{\ell}=1\right)$ and tensor $\left(L_{\ell}=2\right)$ representations of continuous rotations about the $(1,1,1)$ axis, respectively; in this case, since angular momentum $L_{\ell}=3$ represents a state invariant under $C_{3}$ rotations, these are analogous to two vector representations.

For our purposes, it is convenient to describe these irreducible representations using differential operators of the form $\partial_{i} \partial_{j}$. Specifically, we represent the matrix element in the basis of $\partial_{l}^{2},\left(\partial_{\hat{u}_{\perp}}^{2}+\partial_{u}^{2}\right), \partial_{l} \partial_{u}, \partial_{l} \partial_{\hat{u}_{\perp}},\left(\partial_{u}^{2}-\partial_{\hat{u}_{\perp}}^{2}\right), \partial_{u} \partial_{\hat{u}_{\perp}}$ which form irreducible representations of the quadratic derivative operator.

In this notation, we have

$$
\begin{aligned}
\Gamma_{A_{1}} & =\left|\partial_{l}^{2}\right\rangle\left\langle\partial_{l}^{2}\left|, \quad \Gamma_{A_{2}}=\right| \partial_{u}^{2}+\partial_{\hat{u}_{\perp}}^{2}\right\rangle\left\langle\partial_{u}^{2}+\partial_{\hat{u}_{\perp}}^{2}\right|, \\
\Gamma_{a} & =\frac{-1}{2}\left(\left|\partial_{l} \partial_{u}\right\rangle\left\langle\partial_{l} \partial_{u}|-| \partial_{l} \partial_{\hat{u}_{\perp}}\right\rangle\left\langle\partial_{l} \partial_{\hat{u}_{\perp}}\right|\right) \\
& +\frac{\sqrt{3}}{2}\left|\partial_{l} \partial_{\hat{u}_{\perp}}\right\rangle\left\langle\partial_{l} \partial_{u}\left|-\frac{\sqrt{3}}{2}\right| \partial_{l} \partial_{u}\right\rangle\left\langle\partial_{l} \partial_{\hat{u}_{\perp}}\right|, \\
\Gamma_{b} & =\frac{-1}{2}\left(\left|\partial_{u}^{2}-\partial_{\hat{u}_{\perp}}^{2}\right\rangle\left\langle\partial_{u}^{2}-\partial_{\hat{u}_{\perp}}^{2}|+| 2 \partial_{u} \partial_{\hat{u}_{\perp}}\right\rangle\left\langle 2 \partial_{u} \partial_{\hat{u}_{\perp}}\right|\right) \\
& -\frac{\sqrt{3}}{2}\left|2 \partial_{u} \partial_{\hat{u}_{\perp}}\right\rangle\left\langle\partial_{u}^{2}-\partial_{\hat{u}_{\perp}}^{2}\left|+\frac{\sqrt{3}}{2}\right| \partial_{u}^{2}-\partial_{\hat{u}_{\perp}}^{2}\right\rangle\left\langle 2 \partial_{u} \partial_{\hat{u}_{\perp}}\right| .
\end{aligned}
$$

From these, we deduce the form (22) for the operators $D_{i}^{a}, D_{i}^{b}$ transforming in the $2 \mathrm{D}$ irreps $\Gamma_{a}$ and $\Gamma_{b}$.

\section{APPENDIX C: THE CASE $\beta=0$}

As discussed in the main text, taking $D_{i}$ to transform purely in the $\Gamma^{a}$ irreducible representation of $C_{3}$ yields a theory that is reminiscent of a stack of decoupled 2D layers. Here, we give a few more details on the nature of the gauge-invariant operators and mobility of sources in this theory.

The $\Gamma^{a}$ irreducible representation leads to $D_{i}$ operators of the form

$$
D_{1}=d_{\ell} d_{u}, \quad D_{2}=d_{\ell} d_{u_{\perp}}
$$

In this case, the line operator

$$
\oint\left(A_{1} d u+A_{2} d u_{\perp}\right)
$$

is gauge invariant for any closed curve in the $\left(u, u_{\perp}\right)$ plane. The charge in, and consequently dipole moment perpendicular to, each $\left(u, u_{\perp}\right)$ plane is also conserved.

In addition, the line operators

$$
\oint A_{1} d \ell, \quad \oint A_{2} d \ell
$$

are also gauge invariant. Open line operators of this type can be made gauge invariant by binding dipoles along the $u$ and $u_{\perp}$ directions, respectively. However, these are one-dimensional line operators, in the sense that they are not free to bend. To see this, consider

$$
\delta\left(\int_{x}^{y} A_{1} d \ell\right)=\partial_{1} \alpha_{x}^{y}, \quad \delta\left(\int_{x}^{y} A_{2} d \ell\right)=\partial_{2} \alpha_{x}^{y} .
$$

By contrast, we have

$$
\delta\left(\int_{x}^{y} A_{1} d u\right)=\delta\left(\int_{x}^{y} A_{2} d u_{\perp}\right)=\partial_{\ell} \alpha_{x}^{y} .
$$

A similar result holds if we replace $u, u_{\perp}, \ell$ with any three distinct directions. Correspondingly, in three dimensions we also find that $\oint B d \ell=0$, so the charge along and dipole moment perpendicular to each $\ell$ line is also conserved.

If $l_{1}, l_{2}$, and $l_{3}$ are not linearly independent, we can, however, have trivalent junctions between one line in the $l_{1}, l_{2}$ plane and two lines in the $l_{3}$ direction. But, this is redundant since in this case $l_{3}$ lies in the plane spanned by $l_{1}$ and $l_{2}$.

Thus, this theory resembles a stack of decoupled vector gauge theories, but with extra conservation laws pertaining to dipole moments in the $\left(u, u_{\perp}\right)$ plane. We expect objects with a dipole moment along $\ell$ that are free to move in the $\left(u, u_{\perp}\right)$ planes, and objects with dipole moment along $u$ or $u_{\perp}$ can move only in the $\ell$ direction.

We finish by noting that the Chern-Simons term described here does not fully gap this theory. Specifically, in this case we may add a Maxwell term that is of the same order in derivatives as the Chern-Simons term since the operator $b=$ $\partial_{u_{\perp}} A_{1}-\partial u A_{2}$ is gauge invariant. Adding a term of the form $\sum_{i} E_{i}^{2}+b^{2}$, where $E_{i}$ is the electric field defined in Eq. (3), leads to a gapless theory due to the presence of a collective mode with $k_{\ell}=0$ that is not affected by the Chern-Simons constraint.

\section{APPENDIX D: LINE OPERATORS IN THE GAPLESS CHERN-SIMONS THEORY}

The constraint obtained by taking the variation of our Lagrangian with respect to $A_{0}$ is

$$
\frac{s}{2 \pi} \partial_{i} B_{i}+\frac{1}{\sqrt{3} g^{2}} \sum_{i j} \partial_{i} \partial_{j} E_{\ell}=\rho .
$$

In order to calculate the commutators of our line operators with the constraint, it is useful to switch to commutators between fields in momentum space. We have

$$
\begin{aligned}
& {\left[A_{x y}(\mathbf{q}), A_{x z}\left(\mathbf{q}^{\prime}\right)\right]=i k \delta\left(\mathbf{q}+\mathbf{q}^{\prime}\right),} \\
& {\left[A_{x y}(\mathbf{q}), A_{y z}\left(\mathbf{q}^{\prime}\right)\right]=-i k \delta\left(\mathbf{q}+\mathbf{q}^{\prime}\right),}
\end{aligned}
$$


where here

$$
k=-i\left[A_{x y}, A_{x z}\right]=2 \pi /(3 s)
$$

Then,

$$
\begin{aligned}
& {\left[A_{x y}(\mathbf{q}), B_{x}\left(\mathbf{q}^{\prime}\right)\right]=i k q_{y}^{\prime} \delta\left(\mathbf{q}+\mathbf{q}^{\prime}\right),} \\
& {\left[A_{x y}(\mathbf{q}), B_{y}\left(\mathbf{q}^{\prime}\right)\right]=i k q_{x}^{\prime} \delta\left(\mathbf{q}+\mathbf{q}^{\prime}\right),} \\
& {\left[A_{x y}(\mathbf{q}), B_{z}\left(\mathbf{q}^{\prime}\right)\right]=-i k\left(q_{y}^{\prime}+q_{x}^{\prime}\right) \delta\left(\mathbf{q}+\mathbf{q}^{\prime}\right) .}
\end{aligned}
$$

Similarly,

$$
\begin{aligned}
& {\left[A_{x z}(\mathbf{q}), B_{x}\left(\mathbf{q}^{\prime}\right)\right]=i k q_{z}^{\prime} \delta\left(\mathbf{q}+\mathbf{q}^{\prime}\right),} \\
& {\left[A_{x z}(\mathbf{q}), B_{y}\left(\mathbf{q}^{\prime}\right)\right]=-i k\left(q_{z}^{\prime}+q_{x}^{\prime}\right) \delta\left(\mathbf{q}+\mathbf{q}^{\prime}\right),} \\
& {\left[A_{x z}(\mathbf{q}), B_{z}\left(\mathbf{q}^{\prime}\right)\right]=i k q_{x}^{\prime} \delta\left(\mathbf{q}+\mathbf{q}^{\prime}\right)}
\end{aligned}
$$

and

$$
\begin{aligned}
& {\left[A_{y z}(\mathbf{q}), B_{x}\left(\mathbf{q}^{\prime}\right)\right]=-i k\left(q_{y}^{\prime}+q_{z}^{\prime}\right) \delta\left(\mathbf{q}+\mathbf{q}^{\prime}\right),} \\
& {\left[A_{y z}(\mathbf{q}), B_{y}\left(\mathbf{q}^{\prime}\right)\right]=i k q_{z}^{\prime} \delta\left(\mathbf{q}+\mathbf{q}^{\prime}\right),} \\
& {\left[A_{y z}(\mathbf{q}), B_{z}\left(\mathbf{q}^{\prime}\right)\right]=i k q_{y}^{\prime} \delta\left(\mathbf{q}+\mathbf{q}^{\prime}\right) .}
\end{aligned}
$$

From these, we obtain that

$$
\frac{s}{2 \pi}\left[A_{x y}\left(\mathbf{q}^{\prime}\right), q_{i} B_{i}(\mathbf{q})\right]=\frac{i}{3}\left(2 q_{x} q_{y}-q_{x} q_{z}-q_{y} q_{z}\right) \delta\left(\mathbf{q}+\mathbf{q}^{\prime}\right),
$$

where we have used the fact that $k s / 2 \pi=\frac{1}{3}$. Similarly,

$$
\begin{aligned}
& \frac{1}{\sqrt{3} g^{2}}\left[A_{x y}\left(\mathbf{q}^{\prime}\right), \sum_{i j} q_{i} q_{j} E_{\ell}(\mathbf{q})\right] \\
& =\frac{i}{3}\left(q_{x} q_{y}+q_{x} q_{z}+q_{y} q_{z}\right) \delta\left(\mathbf{q}+\mathbf{q}^{\prime}\right) .
\end{aligned}
$$

Combining these, we find that

$$
\begin{aligned}
& {\left[A_{x y}\left(\mathbf{q}^{\prime}\right), \frac{s}{2 \pi} \partial_{i} B_{i}+\frac{1}{\sqrt{3} g^{2}} \sum_{i j} \partial_{i} \partial_{j} E_{\ell}\right]} \\
& \quad=\frac{i}{3}\left(3 q_{x} q_{y}\right) \delta\left(\mathbf{q}+\mathbf{q}^{\prime}\right) .
\end{aligned}
$$

Thus, $A_{x y}$ per se does not commute with the constraint. However, the line operators $\int A_{x y} d x, \int A_{x y} d y$ do. Letting $C$ denote our constraint, we have

$$
\begin{aligned}
& {\left[\int d x A_{x y}(\mathbf{r}), C\left(\mathbf{r}^{\prime}\right)\right]} \\
& =\int d x \int d^{3} \mathbf{q} \int d^{3} \mathbf{q}^{\prime}\left[A_{x y}\left(\mathbf{q}^{\prime}\right), C(\mathbf{q})\right] e^{i\left(\mathbf{q}^{\prime} \cdot \mathbf{r}+\mathbf{q} \cdot \mathbf{r}^{\prime}\right)} \\
& =i \int d x \int d^{3} \mathbf{q} e^{i \mathbf{q} \cdot\left(\mathbf{r}^{\prime}-\mathbf{r}\right)} q_{x} q_{y} \\
& =i \int d x \partial_{y^{\prime}} \partial_{x^{\prime}} \delta\left(\mathbf{r}-\mathbf{r}^{\prime}\right) .
\end{aligned}
$$

If we allow ourselves to interchange the derivatives and the integration, this clearly vanishes since integral is 1 no matter what $x^{\prime}$ is. Although this sounds questionable, I believe that the derivative of the $\delta$ function is defined by integrating by parts under the integral, which would give the same result. (In contrast, the derivative of a $\delta$ function that is not under an integral cannot be defined in this way.) Thus, we conclude that the line operators do commute with the constraint. In general, this result will hold for any line integral of $A_{x y}$ along a curve in the $x y$ plane.
[1] X.-G. Wen, Int. J. Mod. Phys. B 4, 239 (1990).

[2] R. Dijkgraaf and E. Witten, Commun. Math. Phys. 129, 393 (1990).

[3] X.-G. Wen and A. Zee, Phys. Rev. B 46, 2290 (1992).

[4] X.-G. Wen, Phys. Rev. Lett. 90, 016803 (2003).

[5] B. A. Bernevig, T. L. Hughes, and S.-C. Zhang, Science 314, 1757 (2006).

[6] L. Fu, C. L. Kane, and E. J. Mele, Phys. Rev. Lett. 98, 106803 (2007).

[7] X. Chen, Z.-X. Liu, and X.-G. Wen, Phys. Rev. B 84, 235141 (2011).

[8] X. Chen, Z.-C. Gu, Z.-X. Liu, and X.-G. Wen, Science 338, 1604 (2012).

[9] A. Y. Kitaev, Ann. Phys. (NY) 303, 2 (2003).

[10] J. Haah, Phys. Rev. A 83, 042330 (2011).

[11] G. B. Halász, T. H. Hsieh, and L. Balents, Phys. Rev. Lett. 119, 257202 (2017).

[12] S. Vijay, J. Haah, and L. Fu, Phys. Rev. B 94, 235157 (2016).

[13] S. Vijay, J. Haah, and L. Fu, Phys. Rev. B 92, 235136 (2015).

[14] C. Chamon, Phys. Rev. Lett. 94, 040402 (2005).

[15] T. H. Hsieh and G. B. Halász, Phys. Rev. B 96, 165105 (2017).

[16] K. Slagle and Y. B. Kim, Phys. Rev. B 96, 165106 (2017).
[17] W. Shirley, K. Slagle, and X. Chen, Annals Phys. 410, 167922 (2019).

[18] B. Yoshida, Phys. Rev. B 88, 125122 (2013).

[19] H. Ma, E. Lake, X. Chen, and M. Hermele, Phys. Rev. B 95, 245126 (2017).

[20] S. Vijay, arXiv:1701.00762.

[21] K. Slagle and Y. B. Kim, Phys. Rev. B 96, 195139 (2017).

[22] H. Ma, A. T. Schmitz, S. A. Parameswaran, M. Hermele, and R. M. Nandkishore, Phys. Rev. B 97, 125101 (2018).

[23] K. Slagle and Y. B. Kim, Phys. Rev. B 97, 165106 (2018).

[24] W. Shirley, K. Slagle, Z. Wang, and X. Chen, Phys. Rev. X 8, 031051 (2018).

[25] M. Pretko and L. Radzihovsky, Phys. Rev. Lett. 120, 195301 (2018).

[26] H. Ma, M. Hermele, and X. Chen, Phys. Rev. B 98, 035111 (2018).

[27] A. Prem, M. Pretko, and R. Nandkishore, Phys. Rev. B 97, 085116 (2018).

[28] M. Pretko, Phys. Rev. B 95, 115139 (2017).

[29] D. Bulmash and M. Barkeshli, Phys. Rev. B 97, 235112 (2018).

[30] A. Prem, J. Haah, and R. Nandkishore, Phys. Rev. B 95, 155133 (2017).

[31] D. Bulmash and M. Barkeshli, arXiv:1806.01855. 
[32] Y. You, T. Devakul, F. J. Burnell, and S. L. Sondhi, Phys. Rev. B 98, 035112 (2018).

[33] T. Devakul, Y. You, F. Burnell, and S. Sondhi, SciPost Phys. 6, 007 (2019).

[34] Y. You, T. Devakul, F. Burnell, and S. Sondhi, arXiv:1805.09800.

[35] W. Shirley, K. Slagle, and X. Chen, SciPost Phys. 6, 041 (2019)

[36] H. Song, A. Prem, S.-J. Huang, and M. A. Martin-Delgado, Phys. Rev. B 99, 155118 (2019).

[37] D. Bulmash and T. Iadecola, Phys. Rev. B 99, 125132 (2019).

[38] A. Prem, S. Vijay, Y.-Z. Chou, M. Pretko, and R. M. Nandkishore, Phys. Rev. B 98, 165140 (2018).

[39] G. Y. Cho, O. Parrikar, Y. You, R. G. Leigh, and T. L. Hughes, Phys. Rev. B 91, 035122 (2015).

[40] K. Slagle, A. Prem, and M. Pretko, Annals Phys. 410, 167910 (2019).

[41] A. Gromov, Phys. Rev. Lett. 122, 076403 (2019).

[42] M. Pretko, Phys. Rev. B 98, 115134 (2018).

[43] S. Pai and M. Pretko, Phys. Rev. B 97, 235102 (2018).

[44] H. Ma and M. Pretko, Phys. Rev. B 98, 125105 (2018).

[45] M. Pretko, Phys. Rev. B 96, 035119 (2017).

[46] M. Pretko, Phys. Rev. B 96, 115102 (2017).

[47] H. Yan, O. Benton, L. D. Jaubert, and N. Shannon, Phys. Rev. Lett. 124, 127203 (2020).
[48] Y. You and F. von Oppen, Phys. Rev. Res. 1, 013011 (2019).

[49] A. Gromov, Phys. Rev. X 9, 031035 (2019).

[50] T. Hansson, V. Oganesyan, and S. Sondhi, Ann. Phys. (NY) 313, 497 (2004).

[51] D. J. Williamson, Phys. Rev. B 94, 155128 (2016).

[52] D. J. Williamson, Z. Bi, and M. Cheng, Phys. Rev. B 100, 125150 (2019)

[53] M. Pretko, Phys. Rev. B 96, 125151 (2017).

[54] K. Slagle, D. Aasen, and D. Williamson, SciPost Phys. 6, 043 (2019).

[55] S. Pai and M. Hermele, Phys. Rev. B 100, 195136 (2019).

[56] C. Xu and C. Wu, Phys. Rev. B 77, 134449 (2008).

[57] A. Prem, S.-J. Huang, H. Song, and M. Hermele, Phys. Rev. X 9, 021010 (2019).

[58] E. Fradkin and F. A. Schaposnik, Phys. Rev. Lett. 66, 276 (1991).

[59] D. Eliezer and G. Semenoff, Ann. Phys. (NY) 217, 66 (1992).

[60] K. Sun, K. Kumar, and E. Fradkin, Phys. Rev. B 92, 115148 (2015).

[61] E. Witten, Commun. Math. Phys. 137, 29 (1991).

[62] T. Wang, W. Shirley, and X. Chen, Phys. Rev. B 100, 085127 (2019).

[63] W. Shirley, K. Slagle, and X. Chen, SciPost Phys. 6, 015 (2019). 